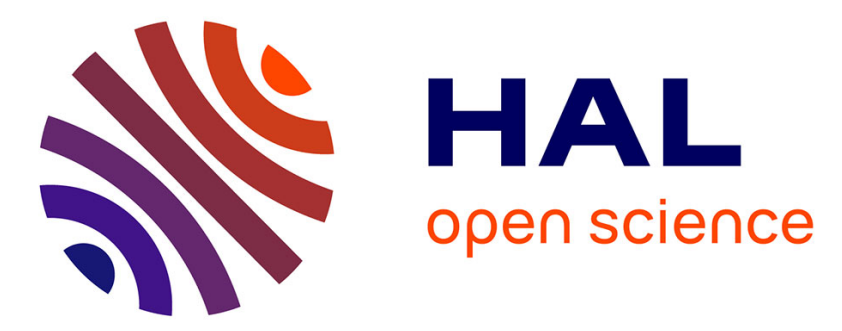

\title{
Parameter maximum likelihood estimation problem for time periodic modulated drift Ornstein Uhlenbeck processes \\ Dominique Dehay
}

\section{- To cite this version:}

Dominique Dehay. Parameter maximum likelihood estimation problem for time periodic modulated drift Ornstein Uhlenbeck processes. Statistical Inference for Stochastic Processes, 2015, 18 (1), pp.6998. 10.1007/s11203-014-9104-7 . hal-00794615v2

\section{HAL Id: hal-00794615 \\ https://hal.science/hal-00794615v2}

Submitted on 16 Apr 2015

HAL is a multi-disciplinary open access archive for the deposit and dissemination of scientific research documents, whether they are published or not. The documents may come from teaching and research institutions in France or abroad, or from public or private research centers.
L'archive ouverte pluridisciplinaire HAL, est destinée au dépôt et à la diffusion de documents scientifiques de niveau recherche, publiés ou non, émanant des établissements d'enseignement et de recherche français ou étrangers, des laboratoires publics ou privés. 


\title{
Parameter maximum likelihood estimation problem for time periodic modulated drift Ornstein Uhlenbeck processes
}

\author{
Dominique Dehay \\ Institut de Recherche Mathématique de Rennes, CNRS umr 6625 \\ Université Rennes 2 \\ email : dominique.dehay@univ-rennes2.fr \\ december 2014
}

\begin{abstract}
In this paper we investigate the large-sample behaviour of the maximum likelihood estimate (MLE) of the unknown parameter $\theta$ for processes following the model

$$
d \xi_{t}=\theta f(t) \xi_{t} d t+d \mathrm{~B}_{t}
$$

where $f: \mathbb{R} \rightarrow \mathbb{R}$ is a continuous function with period, say $P>0$. Here the periodic function $f(\cdot)$ is assumed known. We establish the consistency of the MLE and we point out its minimax optimality. These results comply with the well-established case of an Ornstein Uhlenbek process when the function $f(\cdot)$ is constant. However the case when $\int_{0}^{P} f(t) d t=0$ and $f(\cdot)$ is not identically null presents some special features. For instance in this case whatever is the value of $\theta$, the rate of convergence of the MLE is $T$ as in the case when $\theta=0$ and $\int_{0}^{P} f(t) d t \neq 0$.
\end{abstract}

Keywords Langevin stochastic differential equation. Time-inhomogeneous diffusion process . Periodicity . Ornstein Uhlenbeck process . Maximum likelihood estimator . Local asymptotic minimax bound .

Mathematics Subject Classification (2000) 62 F12 


\section{Introduction}

The non-stationary and seasonal behaviour is quite common for many random phenomena observed in time. This can be due to the influence of external oscillation forces acting on the system under study, or to some internal forces that exist within the system (rotating machinery, waves, cyclical phenomenae, seasonality, time-periodic modulation, etc.). The periodicity may be hidden in the structure of the process, for instance in the covariance structure for a nonstationary periodically correlated process called also cyclostationary signal in signal theory (Gardner et al. 2006), or as in the present paper, in the coefficients of a time-inhomogeneous diffusion process (see also Höpfner and Kutoyants 2010). Many applications of such models can be found in mechanics, communication theory, climatology, econometrics, biology to name but a few (see e.g. Antoni 2009; Chaari et al. 2014; Collet and Martinez 2008; Gardner et al. 2006 ; Serpedin et al. 2005). A large amount of publications have been devoted to discrete time linear models with coefficients which are periodically time-dependent (see e.g. Hurd and Miamee 2007, and references therein). In the present work we consider the continuous-time counterpart of the periodically autoregressive time series model of order 1 (PAR(1) model), introducing a time-periodic modulation in the drift of the Ornstein Uhlenbeck model.

The main purpose of the paper is the maximum likelihood estimation problem for the unknown parameter $\theta \in \mathbb{R}$ in the so called P-periodic Langevin SDE (stochastic differential equation)

$$
d \xi_{t}=\theta f(t) \xi_{t} d t+d \mathrm{~B}_{t}, \quad \xi_{0}, \quad t \geq 0
$$

from the observation of a continuous sample path of the $P$-OU-process $\left\{\xi_{t}, t \geq 0\right\}$. Here $\left\{\mathrm{B}_{t}, t \geq 0\right\}$ is a Brownian motion which is independent with respect to the initial random variable $\xi_{0}$. The time-dependent modulation $f: \mathbb{R} \rightarrow \mathbb{R}$ is some known, no identically null, $P$ periodic and continuous function. Thus the period $P>0$ is known. For simplicity of exposure we have assumed that the diffusion coefficient is equal to 1 . The non-parametric problem of estimation of the modulation function $f(\cdot)$ is out of the scope of the paper and will be subject to another work. Parameter estimation problem for models of SDE with time-dependent drift have been considered by many authors (for instance see Mishra and Prakasa Rao 1985; Liptser and Shiryaev 2001; and recently, Barczy and Pap 2010; Höpfner and Kutoyants 2010 ; Dehling et al. 2010). None considers the case of SDE (1) which cannot be reduced to known models.

Such SDE (1) admits a unique solution for which we exhibit a completely explicit expression (6). This permits us to develop the forthcoming analysis exploiting the periodic structure of the model. In this paper a solution of SDE (1) is called P-periodic Ornstein Uhlenbeck type process and for brevity noted P-OU-process.

In Section 2 we present the main features about this model (Dehay 2014). These properties help us to understand the structure of the model under consideration, and are used in the following sections. The diversity of the statements corresponds to the different recurrence properties of the solution $\left\{\xi_{t}, t \geq 0\right\}$ of SDE (1). More precisely the model possesses a periodic Markov structure which can be described with the help of the associated $P$-segments chain 
$\left\{\mathbf{X}_{n}:=\left\{\xi_{n P+t}, t \in[0, P]\right\}, n \in \mathbb{N}\right\}$ as defined by Höpfner and Kutoyants (2010). According to the signum of $F(P):=\int_{0}^{P} f(t) d t$, the model has a recurrent or transient behaviour. The case where $F(P)=0$ is of particular interest, it corresponds to a null recurrent Markov model. We refer to (Meyn and Tweedie 2009; Revuz 1984) for the notions of recurrent, transient and Harris recurrent Markov chains. For recurrent or transient continuous-time processes we refer to (Has'minskiř 1980 ; Revuz and Yor 1994).

Next in Section 3 we takle with the problem of estimation of the parameter $\theta$ in SDE (1) by the maximum likelihood method, from the observation of a sample path of the process along the finite interval $[0, T]$, as $T \rightarrow \infty$. Liptser and Shiryaev (2001, Theorem 17.2) have given conditions for evaluating the bias and the quadratic error of the maximum likelihood estimator (MLE) of the parameter $\theta$ for a large class of models including the $P$-periodic Langevin SDE (1). Although this result can be applied successfully to model (1) with $f(\cdot) \equiv 1$ (Liptser and Shiryaev 2001, Theorem 17.3), to carry out any checks when $f(\cdot)$ is not constant requires untractable computations. In a recent paper Barczy and Pap (2010) have investigated the MLE for a timeinhomogeneous diffusion process given by the SDE

$$
d \xi_{t}=\theta f(t) \xi_{t} d t+\sigma(t) d \mathrm{~B}_{t}, \quad \xi_{0}, \quad t \geq 0
$$

where $f(\cdot)$ and $\sigma(\cdot)$ are known continuous functions. However due to the periodicity of the function $f(\cdot)$ and so the periodic structure of the process in the present work, their results do not apply to our context except to the Harris recurrent case where $\theta F(P)<0$. In Section 3, thanks to the asymptotic behaviour of the $P$-OU-processes, we establish the strong consistency of the MLE $\widehat{\theta}_{T}$ whatever is the value of $\theta F(P)$ (Theorem 1). We also study the limit law of the scaled deviation $\delta_{T}(\theta)\left(\widehat{\theta}_{T}-\theta\right)$. The normalizing factor $\delta_{T}(\theta)$ as well as the limit law are quite different according to the signum of $\theta F(P)$ (Section 3.3). When $\theta F(P)<0$ or $\theta F(P)>0$ the results are plain generalizations of the classical case where $f(\cdot) \equiv 1$ (Theorems 2 and 5 ). When $F(P)=0$, the limit law of the scaled deviation $T\left(\widehat{\theta}_{T}-\theta\right)$ coincides with the mixed Gaussian law of

$$
\frac{\int_{0}^{1} \mathrm{~B}_{u} d \mathrm{~B}_{u}^{\prime}}{\int_{0}^{1} \mathrm{~B}_{u}^{2} d u}
$$

up to a factor $c(\theta)>0$ whatever is the value of $\theta \in \mathbb{R},\left\{\mathrm{B}_{t}, t \in[0,1]\right\}$ and $\left\{\mathrm{B}_{t}^{\prime}, t \in[0,1]\right\}$ being two independent Brownian motions (Theorem 3), while when $\theta=0$ and $f(\cdot) \equiv 1$, the limit law of $T \widehat{\theta}_{T}$ coincides with the law of

$$
\frac{\int_{0}^{1} \mathrm{~B}_{u} d \mathrm{~B}_{u}}{\int_{0}^{1} \mathrm{~B}_{u}^{2} d u}=\frac{\mathrm{B}_{1}^{2}-1}{2 \int_{0}^{1} \mathrm{~B}_{u}^{2} d u} .
$$

Furthermore we investigate the case when $\theta=0$ and $f(\cdot)$ is not identically null, establishing a link between the results for the two cases $F(P)=0$ versus $\theta=0$ and $f(\cdot) \equiv 1$ (Theorem 4).

Finally Section 4 is devoted to the optimality of the MLE in the sense of local asymptotic minimax property (see Ibragimov and Has'minskǐ̌, 1981; Jeganathan 1995; Le Cam and Yang 1990 ; see also Hájek 1972 ; Le Cam 1969 and 1986). When $\theta F(P)<0$, the recurrence property 
of the P-OU-process entails that the model is locally asymptotically normal (LAN), the scale being $\sqrt{T}$, and the MLE is locally asymptotically minimax for a large class of loss functions (Theorem 6). In the case $\theta F(P) \geq 0$, we establish that the model is locally asymptotically mixed normal (LAMN), and the MLE $\widehat{\theta}_{T}$ has also some local asymptotic optimality (Theorems 7 and 9). Here $\delta_{T}(\theta)=T$ when $\theta F(P)=0$, and $\delta_{T}(\theta)=e^{n \theta F(P)}$ when $\theta F(P)>0, T=n P+t$, $n \in \mathbb{N}, t \in[0, P)$. Moreover the size of the small neighbourhood $\mathcal{U}_{\theta}$ of $\theta$ in the local asymptotic minimax risk (14) is proportional to $\delta_{T}(\theta)^{-1}$ so tends to 0 as $T \rightarrow \infty$ in contrast to the LAN case $\theta F(P)<0$ where in relation (16) the size of $\mathcal{U}_{\theta}$ does not depend on $T$. For the specific quadratic loss function, when $\theta F(P)=0$ we deal with van Trees inequality to establish a Bayesian version of the Cramér-Rao lower bound (Gill and Levit 1995) and to state that the MLE is locally asymptotically minimax with the size of the small neighbourhood $\mathcal{U}_{\theta}$ that does not depend on $T$ as in the LAN case $\theta F(P)<0$ (Theorem 8).

For an easier reading and understanding of the statements of the paper, the proofs are collected in Section 5 .

\section{Background : Time periodic Ornstein Uhlenbeck process}

Here we present some useful properties of the solution of the following P-periodic Langevin SDE

$$
d \xi_{t}=f(t) \xi_{t} d t+d \mathrm{~B}_{t}, \quad \xi_{0}, \quad t \geq 0 .
$$

As the modulation function $f: \mathbb{R} \rightarrow \mathbb{R}$ is periodic and continuous, the usual conditions for the existence and the unicity of the strong solution of SDE when the initial value is fixed, are satisfied (Liptser and Shiryaev 2001). Moreover the strong solution can be expressed easily

$$
\xi_{t}=e^{F(t)}\left(\xi_{0}+\int_{0}^{t} e^{-F(u)} d \mathrm{~B}_{u}\right)
$$

where $F(t):=\int_{0}^{t} f(u) d u$. Remark that as the modulation function $f(\cdot)$ is $P$-periodic, $F(n P+$ $t)=n F(P)+F(t)$ for any $t>0$ and any integer $n$.

The process $\left\{\xi_{t}, t \geq 0\right\}$ is inhomogeneous Markovian whose transition probability density $p_{s, t}(x, \cdot)$ coincides with the density of the normal law $\mathcal{N}\left(x e^{F(t)-F(s)}, \int_{s}^{t} e^{2(F(t)-F(u))} d u\right)$. Notice that the transition probability density is periodic in time $: p_{s+P, t+P}(x, y)=p_{s, t}(x, y)$, for all $s, t, x$ and $y$. Furthermore for each $t \in[0, P]$ the chain $\left\{\xi_{n P+t}\right\}_{n \in \mathbb{N}}$ is homogeneous Markovian, and can be decomposed as

$$
\xi_{n P+t}=e^{F(P)} \xi_{(n-1) P+t}+e^{F(P)+F(t)} \int_{t}^{P} e^{-F(u)} d \mathrm{~B}_{u}^{((n-1) P)}+e^{F(t)} \int_{0}^{t} e^{-F(u)} d \mathrm{~B}_{u}^{(n P)}
$$

where the processes $\mathrm{B}^{(n P)}:=\left\{\mathrm{B}_{n P+u}-\mathrm{B}_{n P}, u \in[0, P]\right\}, n \in \mathbb{N}$, are independent Brownian motions on $[0, P]$. Following Höpfner and Kutoyants (2010, Section 2) we define the $P$-segments sequence $\left\{\mathbf{X}_{n}\right\}_{n \in \mathbb{N}}, \mathbf{X}_{n}:=\left\{\xi_{n P+t}, t \in[0, P]\right\}$. Then the previous decomposition can be rewritten as

$$
\mathbf{X}_{n}(t)=e^{F(P)} \mathbf{X}_{n-1}(t)+e^{F(P)+F(t)}\left(\mathbf{Z}_{n-1}(P)-\mathbf{Z}_{n-1}(t)\right)+e^{F(t)} \mathbf{Z}_{n}(t)
$$


where

$$
\mathbf{Z}_{n}(t):=\int_{0}^{t} e^{-F(u)} d \mathrm{~B}_{u}^{(n P)} .
$$

The sequence $\left(\mathbf{X}_{n}\right)_{n \in \mathbb{N}}$ is a homogeneous Markov chain with state space $\mathcal{C}[0, P]$, the space of real-valued continuous functions defined in $[0, P]$ endowed with the uniform distance. Moreover $\mathcal{L}\left[\mathbf{Z}_{n}(t)\right]=\mathcal{N}(0, G(t))$, where $G(t):=\int_{0}^{t} e^{-2 F(u)} d u$ for any $t$.

For $F(P) \leq 0$, that is $e^{F(P)} \leq 1$, the $P$-segments Markov chain $\left(\mathbf{X}_{n}\right)_{n \in \mathbb{N}}$ is Harris recurrent, more precisely there is a positive measure $\lambda$ on $\left(\mathcal{C}[0, P], \mathbf{C}_{P}\right)$ such that the sets of positive $\lambda$ measure are visited infinitely often whatever is the initial state $\mathbf{X}_{0} \in \mathcal{C}[0, P]$. Here $\mathbf{C}_{P}$ denotes the Borel $\sigma$-field of the separable metric space $\mathcal{C}[0, P]$.

When $F(P)<0$, this positive measure $\lambda$ can be chosen as the limit probability law given in limit (3) below and it is invariant for the Markov chain $\left(\mathbf{X}_{n}\right)_{n \in \mathbb{N}}$. Thus this Harris recurrent Markov chain is positive, that is for any $A \in \mathbf{C}_{P}$ such that $\lambda[A]>0$, the expectation of the amount of steps between two visits in $A$ is finite (Meyn and Tweedie 1993, Theorem 10.4.9). Furthermore the Markov chain $\left(\mathbf{X}_{n}\right)_{n \in \mathbb{N}}$ fulfils some ergodicity properties (Höpfner and Kutoyants 2010, Theorem 2.1) and

$$
\lim _{n \rightarrow \infty} \mathcal{L}\left[\mathbf{X}_{n}\right]=\lim _{n \rightarrow \infty} \mathcal{L}\left[\xi_{n P+} \cdot\right]=\mathcal{L}\left[e^{F(\cdot)}\left(\zeta_{1}+\mathbf{Z}\right)\right] \quad \text { in } \mathcal{C}[0, P] .
$$

where $\zeta_{1}$ is a real-valued Gaussian variable $\mathcal{N}\left(0, \frac{G(P)}{e^{-2 F(P)}-1}\right), \mathbf{Z}:=\{\mathbf{Z}(t), t \in[0, P]\}$ is a Gausssian process with representation $\mathbf{Z}(t)=\int_{0}^{t} e^{-F(u)} d \mathrm{~B}_{u}^{\prime},\left\{\mathrm{B}_{u}^{\prime}, u \in[0, P]\right\}$ is a Brownian motion on $[0, P]$, independent with respect to the random variable $\zeta_{1}$.

When $F(P)=0$, then the Harris recurrent chain $\left(\mathbf{X}_{n}\right)_{n \in \mathbb{N}}$ admits a $\sigma$-finite (unbounded) invariant measure, so it is null Harris recurrent and for any $A \in \mathbf{C}_{P}$ such that $0<\lambda[A]<\infty$, the expectation of the amount of steps between two visits in $A$ is infinite. Notice that in this case for each $t \in[0, P]$, the Markov chain $\left\{\xi_{n P+t}, n \in \mathbb{N}\right\}$ is a random walk on $\mathbb{R}$. Furthermore

$$
\lim _{n \rightarrow \infty} \mathcal{L}\left[\frac{\mathbf{X}_{n}}{\sqrt{n}}\right]=\lim _{n \rightarrow \infty} \mathcal{L}\left[\frac{\xi_{n P+\cdot}}{\sqrt{n}}\right]=\mathcal{L}\left[e^{F(\cdot)} \zeta_{2}\right] \quad \text { in } \mathcal{C}[0, P]
$$

where $\zeta_{2}$ is a real-valued Gaussian variable $\mathcal{N}(0, G(P))$. Then $\xi_{T} / \sqrt{T}$ converges in law to the Gaussian law $\mathcal{N}(0, G(P) / P)$.

If $F(P)>0$, the $P$-segments chain $\mathbf{X}$ and the $P$-OU process $\left\{\xi_{t}, t \geq 0\right\}$ are transient (Meyn and Tweedie 1993, Theorem 8.0.1; Has'minskiĩ 1980, Chap.IV.2 pp.113). Moreover

$$
\lim _{n \rightarrow \infty} e^{-n F(P)} \mathbf{X}_{n}=\lim _{n \rightarrow \infty} e^{-n F(P)} \xi_{n P+\cdot}=e^{F(\cdot)}\left(\xi_{0}+\zeta_{3}\right) \quad \text { a.e. in } \mathcal{C}[0, P]
$$

where $\zeta_{3}$ is a real-valued Gaussian variable $\mathcal{N}\left(0, \frac{G(P)}{1-e^{-2 F(P)}}\right), \zeta_{3}$ and $\xi_{0}$ being independent. Thus $e^{-F(T)} \xi_{T}$ converges a.e. to $\xi_{0}+\zeta_{3}$ as $T \rightarrow \infty$.

\section{Parameter estimation}

Henceforth we deal with the parameter estimation problem for model (1). Consider a diffusion process observed on the interval $[0, T]$ following the $P$-periodic Langevin SDE (1) with 
parameter $\theta \in \mathbb{R}$ and with initial variable $\xi_{0}$ independent with respect to $\left\{\mathrm{B}_{t}, t \geq 0\right\}$ and on the parameter $\theta$ for simplicity of exposure. Then we turn to the problem of estimating the unknown parameter $\theta \in \mathbb{R}$ on the basis of the observation $\left\{\xi_{t}, t \in[0, T]\right\}$. More precisely we study the asymptotic behaviour of the maximum likelihood estimator (MLE) of $\theta$ as the observation time $T$ goes to infinity.

Throughout the following we assume that the modulation function $f(\cdot)$ is any periodic continuous function with period $P>0$, except for specified cases. Then the strong solution of the $P$-periodic SDE (1) exists, is unique and admits an explicit expression

$$
\xi_{t}:=\xi_{t}^{(\theta)}=e^{\theta F(t)} \xi_{0}+\int_{0}^{t} e^{\theta(F(t)-F(u))} d \mathrm{~B}_{u},
$$

for $t \in[0, \infty)$. Notice that $\xi_{t}^{(0)}=\xi_{0}+\mathrm{B}_{t}$. For simplication we write $\xi_{t}$ for $\xi_{t}^{(\theta)}$ when there is no possibility of confusion. Next in the notations $\mathrm{P}_{\theta}, \mathrm{E}_{\theta}$ and $\mathcal{L}_{\theta}$, the index indicates that we consider that the true value of the parameter is $\theta$. We need also the following functions

$$
G_{\theta}(t):=\int_{0}^{t} e^{-2 \theta F(u)} d u \quad \text { and } \quad H_{\theta}(t):=\int_{0}^{t} f(u)^{2} e^{2 \theta F(u)} d u
$$

for $t \in[0, \infty)$ and $\theta \in \mathbb{R}$.

\subsection{MLE for Ornstein Uhlenbeck processes}

First we recall some well-established results on the maximum likelihood parameter estimation for Ornstein Uhlenbeck processes. Assume that the modulation function $f(\cdot)$ is identically equal to $1, f(\cdot) \equiv 1$. The problem of estimation of the parameter $\theta$ for this model have been subject to a very large amount of contributions. In this classical case the MLE of $\theta$ is equal to

$$
\widehat{\theta}_{T}=\frac{\int_{0}^{T} \xi_{u} d \xi_{u}}{\int_{0}^{T} \xi_{u}^{2} d u}=\theta+\frac{\int_{0}^{T} \xi_{u} d \mathrm{~B}_{u}}{\int_{0}^{T} \xi_{u}^{2} d u}
$$

and its behaviour as $T \rightarrow \infty$ is well-known (see e.g. Basawa and Scott 1983; Bishwal 2008; Brown and Hewitt 1975; Feigin 1979; Kutoyants 2004; Liptser and Shiryaev 1977).

(i) For $\theta<0$, the process $\left\{\xi_{t}, t \geq 0\right\}$ is positive recurrent, ergodic with invariant measure $\mathcal{N}(0,1 /(2|\theta|))$, and

$$
\lim _{T \rightarrow \infty} \mathcal{L}_{\theta}\left[\sqrt{T}\left(\widehat{\theta}_{T}-\theta\right)\right]=\mathcal{N}(0,2|\theta|) .
$$

(ii) For $\theta=0$, the process $\left\{\xi_{t}, t \geq 0\right\}$ is null recurrent

$$
\lim _{T \rightarrow \infty} \mathcal{L}_{0}\left[T \widehat{\theta}_{T}\right]=\mathcal{L}\left[\frac{\int_{0}^{1} \mathrm{~B}_{t} d \mathrm{~B}_{t}}{\int_{0}^{1} \mathrm{~B}_{t}^{2} d t}\right]=\mathcal{L}\left[\frac{\mathrm{B}_{1}^{2}-1}{2 \int_{0}^{1} \mathrm{~B}_{t}^{2} d t}\right] .
$$

(iii) For $\theta>0$, the process is transient $:\left|\xi_{t}\right| \rightarrow \infty$ with probability 1

$$
\lim _{T \rightarrow \infty} \mathcal{L}_{\theta}\left[\frac{e^{\theta T}}{\sqrt{2 \theta}}\left(\widehat{\theta}_{T}-\theta\right)\right]=\mathcal{L}\left[\frac{\nu}{\xi_{0}+\zeta^{(\theta)}}\right]
$$

on $\left\{\xi_{0}+\zeta^{(\theta)} \neq 0\right\}$, where $\mathcal{L}[\nu]=\mathcal{N}(0,1)$ and $\mathcal{L}\left[\zeta^{(\theta)}\right]=\mathcal{N}(0,1 /(2 \theta))$. The random variables $\xi_{0}, \nu$ and $\zeta^{(\theta)}$ are independent. 


\subsection{MLE for $P$-OU processes}

Now we consider the general $P$-periodic model (1). As the function $f(\cdot)$ is continuous and almost every sample path of the process $\left\{\xi_{t}, t \geq 0\right\}=\left\{\xi_{t}^{(\theta)}, t \geq 0\right\}$ is also continuous we have

$$
\int_{0}^{T} f(u)^{2} \xi_{u}^{2} d u<\infty \quad \mathrm{P}_{\theta} \text {-a.e. }
$$

for any $\theta \in \mathbb{R}$ and any $T>0$. Thus

$$
\mathrm{P}_{\theta}\left[\int_{0}^{T} f(u)^{2} \xi_{u}^{2} d u<\infty\right]=\mathrm{P}_{0}\left[\int_{0}^{T} f(u)^{2}\left(\xi_{0}+\mathrm{B}_{u}\right)^{2} d u<\infty\right]=1 .
$$

Thanks to (Liptser and Shiryaev 2001, Theorem 7.7 and formula (17.24)) the law $\mathrm{P}_{\theta}^{T}$ of $\left\{\xi_{t}=\right.$ $\left.\xi_{t}^{(\theta)}, t \in[0, T]\right\}$ is absolutely continuous with respect to the law $\mathrm{P}_{0}^{T}$ of $\left\{\xi_{t}^{(0)}=\xi_{0}+\mathrm{B}_{t}, t \in[0, T]\right\}$, and the log-likelihood ratio of $\mathrm{P}_{\theta}^{T}$ to $\mathrm{P}_{0}^{T}$ verifies

$$
\Lambda_{T}^{(\theta)}=\theta \int_{0}^{T} f(u) \xi_{u} d \xi_{u}-\frac{\theta^{2}}{2} \int_{0}^{T} f(u)^{2} \xi_{u}^{2} d u=\theta \int_{0}^{T} f(u) \xi_{u} d \mathrm{~B}_{u}+\frac{\theta^{2}}{2} \int_{0}^{T} f(u)^{2} \xi_{u}^{2} d u .
$$

Since

$$
\int_{0}^{T} f(u)^{2} \xi_{u}^{2} d u>0 \quad \mathrm{P}_{\theta^{-} \text {a.e., }}
$$

the maximum likelihood estimator $\widehat{\theta}_{T}$ of $\theta$ is well defined and equal to

$$
\widehat{\theta}_{T}=\frac{\int_{0}^{T} f(u) \xi_{u} d \xi_{u}}{\int_{0}^{T} f(u)^{2} \xi_{u}^{2} d u}=\theta+\frac{\int_{0}^{T} f(u) \xi_{u} d \mathrm{~B}_{u}}{\int_{0}^{T} f(u)^{2} \xi_{u}^{2} d u} .
$$

\subsection{Consistency of the MLE}

Assume $\theta \in \mathbb{R}$ be fixed. Let

$$
U_{t}:=\int_{0}^{t} f(u) \xi_{u} d \mathrm{~B}_{u}
$$

and let $\left\{\mathcal{F}_{t}, t \geq 0\right\}$ be the filtration generated by $\xi_{0}$ and the Brownian motion $\left\{\mathrm{B}_{t}, t \geq 0\right\}$. Then $\left\{U_{t}, t \geq 0\right\}$ is a zero-mean continuous martingale with respect to $\left\{\mathcal{F}_{t}, t \geq 0\right\}$. Its quadratic variation is equal to

$$
J_{t}:=\int_{0}^{t} f(u)^{2} \xi_{u}^{2} d u
$$

and

$$
\mathrm{E}_{\theta}\left[U_{t}^{2}\right]=\mathrm{E}_{\theta}\left[J_{t}\right]=\int_{0}^{t} f(u)^{2} \mathrm{E}_{\theta}\left[\xi_{u}^{2}\right] d u<\infty .
$$

The strong law of large numbers for martingales (see Liptser and Shiryaev 2001, Lemma 17.4) entails that

$$
\lim _{T \rightarrow \infty} J_{T}^{-1} U_{T}=0 \quad \mathrm{P}_{\theta^{-}} \text {-a.e. on }\left\{J_{\infty}=\infty\right\}
$$

where $J_{\infty}:=\lim _{T \rightarrow \infty} J_{T}$. Hence to prove the strong consistency of the MLE $\widehat{\theta}_{T}$ it suffices to show that $\mathrm{P}_{\theta}\left[J_{\infty}<\infty\right]=0$. This is done thanks to the asymptotic results in Section 2. 
Theorem 1 For any $\theta \in \mathbb{R}$, the $M L E \widehat{\theta}_{T}$ is strongly consistent

$$
\lim _{T \rightarrow \infty} \widehat{\theta}_{T}=\theta \quad \text { P-a.e. }
$$

Hence the MLE $\widehat{\theta}_{T}$ is a consistent estimator of $\theta$

$$
\lim _{T \rightarrow \infty} \mathrm{P}_{\theta}\left[\left|\widehat{\theta}_{T}-\theta\right|>\epsilon\right]=0
$$

for any $\epsilon>0$, whatever is the value of $F(P)$. Actually, in the following we will see that this consistency is uniform with respect to $\theta$ varying in any compact set contained in $\left\{\theta^{\prime} \in \mathbb{R}\right.$ : $\left.\theta^{\prime} F(P)<0\right\}$ or in $\left\{\theta^{\prime} \in \mathbb{R}: \theta^{\prime} F(P)>0\right\}$ if $F(P) \neq 0$, and in any compact subset of $\mathbb{R}$ if $F(P)=0$, the function $f(\cdot)$ being not identically null.

Next we deal with the asymptotic behaviour of the deviation $\widehat{\theta}_{T}-\theta$ according to the signum of $\theta F(P)$.

\subsubsection{Case $\theta F(P)<0$}

When $\theta F(P)<0$, the ergodicity and recurrent properties of the $P$-OU process $\left\{\xi_{t}, t \geq 0\right\}$ imply that the maximum likelihood estimator $\widehat{\theta}_{T}$ has a standard asymptotic behaviour. Thus the result complies with the well-known case of the stationary Ornstein Uhlenbeck processes.

Theorem 2 Let $K_{-}$be any compact subset of $\mathbb{R}$ contained in $\{\theta: \theta F(P)<0\}$. Then

$$
\lim _{T \rightarrow \infty} \mathcal{L}_{\theta}\left[\sqrt{J_{T}}\left(\widehat{\theta}_{T}-\theta\right)\right]=\mathcal{N}(0,1)
$$

and

$$
\lim _{T \rightarrow \infty} \mathcal{L}_{\theta}\left[\sqrt{T}\left(\widehat{\theta}_{T}-\theta\right)\right]=\mathcal{N}\left(0, I(\theta)^{-1}\right)
$$

uniformly with respect to $\theta$ varying in $K_{-}$. The Fisher information $I(\theta)$ is equal to

$$
I(\theta):=\frac{G_{\theta}(P) H_{\theta}(P)}{P\left(e^{-2 \theta F(P)}-1\right)}+\frac{1}{P} \int_{0}^{P} f(u)^{2} e^{2 \theta F(u)} G_{\theta}(u) d u>0 .
$$

\subsubsection{Case $\theta F(P)=0$}

When $\theta F(P)=0$, the Markov $P$-OU process $\left\{\xi_{t}, t \geq 0\right\}$ has a null recurrent behaviour. The maximum likelihood estimator $\widehat{\theta}_{T}$ is consistent. In the following theorem, we see that the rate of convergence is $T$ and the asymptotic law of the scaled deviation $T\left(\widehat{\theta}_{T}-\theta\right)$ is not standard. However using the random scale $\sqrt{J_{T}}$ instead of $T$, the limit law is parameter free : it does not depend on the model.

Theorem 3 Assume that $F(P)=0$ and the function $f(\cdot)$ is not identically null. Then

$$
\lim _{T \rightarrow \infty} \mathcal{L}_{\theta}\left[\sqrt{J_{T}}\left(\widehat{\theta}_{T}-\theta\right)\right]=\mathcal{N}(0,1)
$$


and

$$
\lim _{T \rightarrow \infty} \mathcal{L}_{\theta}\left[T\left(\widehat{\theta}_{T}-\theta\right)\right]=\mathcal{L}\left[\frac{P}{\sqrt{G_{\theta}(P) H_{\theta}(P)}} \frac{\zeta}{\sqrt{\int_{0}^{1} \mathrm{~B}_{u}^{2} d u}}\right]
$$

where the real-valued random variable $\zeta$ is Gaussian $\mathcal{N}(0,1)$ and is independent with respect to the Brownian motion $\left\{\mathrm{B}_{t}, t \in[0,1]\right\}$. The limits being uniform with respect to $\theta$ varying in any compact subset of $\mathbb{R}$.

It is worth to notice that in Theorem 3 when $F(P)=0$ and $f(\cdot)$ not identically null we introduce a Gaussian variable $\zeta$ independent with respect to the Brownian motion in order to define the limit law, whatever is the value of $\theta \in \mathbb{R}$. While for $\theta=0$ and $f(\cdot) \equiv 1$, so $F(P) \neq 0$, we have the well-established limit (7) expressed with only one Brownian motion. With the following theorem, we point out that this limit (7) for $\theta=0$ can be seen as a particular case of the more general case where $f(\cdot)$ is any periodic continuous non identically null function. We recall that here the basic model is the $P$-periodic Langevin SDE (1) and the MLE $\widehat{\theta}_{T}$ is defined by relation (9).

Theorem 4 Assume that $\theta=0$ and the function $f(\cdot)$ is not identically null. Then

$$
\lim _{T \rightarrow \infty} \mathcal{L}_{0}\left[T \widehat{\theta}_{T}\right]=\mathcal{L}\left[\frac{\sqrt{P H_{0}(P)-F(P)^{2}}}{H_{0}(P)} \frac{\zeta}{\sqrt{\int_{0}^{1} \mathrm{~B}_{u}^{2} d u}}+\frac{F(P)}{2 H_{0}(P)} \frac{\left(\mathrm{B}_{1}^{2}-1\right)}{\int_{0}^{1} \mathrm{~B}_{u}^{2} d u}\right]
$$

where the random variable $\zeta$ is Gaussian $\mathcal{N}(0,1)$, and is independent with respect to the Brownian motion $\left\{\mathrm{B}_{t}, t \in[0,1]\right\}$. Recall that $F(P)=\int_{0}^{P} f(u) d u$ and $H_{0}(P)=\int_{0}^{P} f(u)^{2} d u$.

Thus, when $F(P)=0$ and the function $f(\cdot)$ is not identically null, we obtain the limit law stated in Theorem 3 with $\theta=0$. When the function $f(\cdot)$ is constant non null, Theorem 4 reduces to the classical usual limit (7).

\subsubsection{Transient case : $\theta F(P)>0$}

The following result generalizes the well-known case when $f(\cdot) \equiv 1$ and $\theta>0$. The random local scale $\sqrt{J_{T}}$ gives a parameter free limit law.

Theorem 5 Let $K_{+}$be any compact subset of $\mathbb{R}$ contained in $\{\theta: \theta F(P)>0\}$. Then

$$
\lim _{T \rightarrow \infty} \mathcal{L}_{\theta}\left[\sqrt{J_{T}}\left(\widehat{\theta}_{T}-\theta\right)\right]=\mathcal{N}(0,1)
$$

and

$$
\lim _{n \rightarrow \infty} \mathcal{L}_{\theta}\left[K_{\theta}(\cdot) e^{n \theta F(P)}\left(\widehat{\theta}_{n P+\cdot}-\theta\right)\right]=\mathcal{L}\left[\frac{\zeta}{\xi_{0}+\zeta_{3}^{(\theta)}}\right] \quad \text { in } \mathcal{C}[0, P]
$$

uniformly with respect to $\theta$ varying in the compact set $K_{+}$. The real-valued random variable $\zeta$ is Gaussian $\mathcal{N}(0,1)$ and $\zeta_{3}^{(\theta)}$ is Gaussian $\mathcal{N}\left(0, \frac{G_{\theta}(P)}{1-e^{-2 \theta F(P)}}\right)$. Moreover $\xi_{0}, \zeta$ and $\zeta_{3}^{(\theta)}$ are independent. Here

$$
K_{\theta}(t):=\sqrt{\frac{H_{\theta}(P)}{e^{2 \theta F(P)}-1}+H_{\theta}(t)} .
$$


Notice that the function $(\theta, t) \mapsto K_{\theta}(t)$ is positive, continuous and $K_{\theta}(P)=e^{\theta F(P)} K_{\theta}(0)$, and recall that $F(n P+t)=n F(P)+F(t)$. Thus, denoting $T_{P}:=T$ modulo $P$, Theorem 5 implies that $K_{\theta}\left(T_{P}\right) e^{\theta F(T)}\left(\widehat{\theta}_{T}-\theta\right)$ converges in law to $\zeta /\left(\xi_{0}+\zeta_{3}^{(\theta)}\right)$ uniformly with respect to $\theta$ varying in the compact set $K_{+}$as $T \rightarrow \infty$.

Whenever $\xi_{0}=0$, limit law (13) is the Cauchy law with density function $x \mapsto \frac{c_{\theta}}{\pi\left(c_{\theta}^{2}+x^{2}\right)}$ where $c_{\theta}=\sqrt{\frac{1-e^{-2 \theta F(P)}}{G_{\theta}(P)}}$.

\section{Optimality}

Now we takle the problem of optimality for the maximum likelihood estimator $\widehat{\theta}_{T}$. When the size of the sample path observation $T$ is large, we would like to know how good or optimal is the maximum likelihood procedure for the estimation of the parameter $\theta$. In the following we denote by $\mathrm{P}_{\theta}^{T}$, the law of the observed process $\left\{\xi_{t}, t \in[0, T]\right\}$ when the value of the parameter is $\theta$. Recall that for simplicity we assume that the law of the random variable $\xi_{0}$ does not depend on the parameter $\theta$.

We consider here the asymptotic optimality in the sense of local asymptotic minimax lower bound of the risk of $\left\{\bar{\theta}_{T}\right\}:=\left\{\bar{\theta}_{T}, T>0\right\}$ for the estimation of $\theta$, that is

$$
R_{\theta}\left(\left\{\bar{\theta}_{T}\right\}\right):=\lim _{\mathcal{U}_{\theta}} \liminf _{T \rightarrow \infty} \sup _{\theta^{\prime} \in \mathcal{U}_{\theta}} \mathrm{E}_{\theta^{\prime}}\left[L\left(\delta_{T}\left(\theta^{\prime}\right)^{-1}\left(\bar{\theta}_{T}-\theta^{\prime}\right)\right)\right]
$$

where $\bar{\theta}_{T}$ is any statistic function of the observation $\left\{\xi_{t}, t \in[0, T]\right\}$. Here $\mathcal{U}_{\theta}$ is some neighbourhood of $\theta$ which decreases to $\{\theta\}$ in some way depending on the model, $\delta_{T}(\theta)$ is some positive coefficient called local scale, and $L(\cdot)$ is a loss function in $\mathbb{R}, L(\cdot) \in \mathcal{L}, \mathcal{L}$ being the set of Borel functions $L: \mathbb{R} \rightarrow[0, \infty)$ symmetric, continuous at 0 with $L(0)=0$, and non-decreasing on $[0, \infty)$ (see Hájek 1972; Ibragimov and Has'minskiř 1981, pp.18-19; Le Cam and Yang 1990 ; see also Jeganathan 1995, pp.838). Notice that the set of discontinuity of any bounded loss function $L \in \mathcal{L}$ is at most countable. Clearly all functions $L(x)=|x|^{p}, p>0$, as well as $L(x)=\mathbf{1}(x>a)$, $a>0$, belong to $\mathcal{L}$. (Here $\mathbf{1}(x>a)$ denotes the indicator function.)

Let $\theta \in \mathbb{R}$ be fixed, and let $\delta_{T}(\theta) \rightarrow 0$ as $T \rightarrow \infty$. Considering local optimality, denote by $\Lambda_{T}^{(\theta, u)}$ the log-likelihood ratio function of the law $\mathrm{P}_{\theta+u \delta_{T}(\theta)}^{T}$ of $\left\{\xi_{t}^{\left(\theta+u \delta_{T}^{(\theta))}\right.}, t \in[0, T]\right\}$ with respect to the law $\mathrm{P}_{\theta}^{T}$ of $\left\{\xi_{t}^{(\theta)}, t \in[0, T]\right\}$, these laws being equivalent. Thanks to relation (8), we have

$$
\Lambda_{T}^{(\theta, u)}:=\ln \left(\frac{d \mathrm{P}_{\theta+u \delta_{T}(\theta)}^{T}}{d \mathrm{P}_{\theta}^{T}}\right)\left(\xi^{(\theta)}\right)=u \delta_{T}(\theta) U_{T}-\frac{1}{2} u^{2} \delta_{T}(\theta)^{2} J_{T} .
$$

In the light of the previous results, it is not surprising to see in the following that the loglikelihood ratio $\Lambda_{T}^{(\theta, u)}$ has a quite different behaviour according to the signum of $\theta F(P)$. 


\subsection{Case $\theta F(P)<0$}

When $\theta F(P)<0$, thanks to Theorem 2 we take the local scale $\delta_{T}(\theta):=T^{-1 / 2}$. Then the log-likelihood ratio $\Lambda_{T}^{(\theta, u)}$ can be decomposed as

$$
\Lambda_{T}^{(\theta, u)}=u \Delta_{T}^{(\theta)}-\frac{1}{2} u^{2} I(\theta)+r_{T}(\theta, u)
$$

where $\Delta_{T}^{(\theta)}:=T^{-1 / 2} U_{T}$ is asymptotically normal

$$
\lim _{T \rightarrow \infty} \mathcal{L}_{\theta}\left[\Delta_{T}^{(\theta)}\right]=I(\theta)^{1 / 2} \mathcal{N}(0,1)
$$

the Fisher information $I(\theta)$ is defined by relation (12) and

$$
\mathrm{P}_{\theta}-\lim _{T \rightarrow \infty} r_{T}(\theta, u)=0
$$

uniformly with respect to $\theta$ varying in any compact subset of $\{\theta: \theta F(P)<0\}$. Thus the family of laws $\left\{\mathrm{P}_{\theta}^{T}: \theta F(P)<0\right\}, T>0$, is LAN (locally asymptotically normal) at each point $\theta \in \mathbb{R}$ such that $\theta F(P)<0$ (see e.g. Hájek 1972; Ibragimov and Has'minskiř 1981; Le Cam and Yang 1990). Then the local asymptotic lower bound result (Hájek 1972, Theorem 4.1; Ibragimov and Has'minskiǐ (1981), Theorem II.12.1) entails that

$$
\lim _{\epsilon \rightarrow 0} \liminf _{T \rightarrow \infty} \sup _{\left|\theta-\theta^{\prime}\right|<\epsilon} \mathrm{E}_{\theta^{\prime}}\left[L\left(\sqrt{T}\left(\bar{\theta}_{T}-\theta^{\prime}\right)\right)\right] \geq \frac{1}{\sqrt{2 \pi}} \int_{\mathbb{R}} L\left(y I(\theta)^{-1 / 2}\right) e^{-y^{2} / 2} d y
$$

for any loss function $L(\cdot) \in \mathcal{L}$, and for any family $\left\{\bar{\theta}_{T}, T>0\right\}$ of estimators of $\theta$, that is $\bar{\theta}_{T}$ is measurable with respect to the observation $\left\{\xi_{t}, t \in[0, T]\right\}$ (see also Le Cam and Yang 1990, Section 5.6 Theorem 1). Notice that in this case the small neighbourhood $\mathcal{U}_{\theta}$ of $\theta$ in the expression (14) of the local asymptotic minimax risk, is $\left\{\theta^{\prime}:\left|\theta^{\prime}-\theta\right| \leq \epsilon\right\}$ and its size $2 \epsilon>0$ does not depend on $T$. Besides Theorem 2 gives

$$
\lim _{T \rightarrow \infty} \mathcal{L}_{\theta}\left[\sqrt{T}\left(\widehat{\theta}_{T}-\theta\right)\right]=I(\theta)^{-1 / 2} \mathcal{N}(0,1)
$$

uniformly with respect to $\theta$ varying in any compact subset of $\{\theta: \theta F(P)<0\}$. So thanks to the convergence result from Ibragimov and Has'minskiř (1981, Appendix I Theorem 8) the next statement follows immediately.

Theorem 6 Assume that $F(P) \neq 0$. Then the $M L E \widehat{\theta}_{T}$ is locally asymptotically minimax with local scale $T^{-1 / 2}$ at any $\theta$ such that $\theta F(P)<0$ and for any bounded loss function $L(\cdot) \in \mathcal{L}$, in the sense that its local asymptotic minimax risk $R_{\theta}(\widehat{\theta})$ is equal to the lower bound right hand side of inequality (16)

$$
\lim _{\epsilon \rightarrow 0} \lim _{T \rightarrow \infty} \sup _{\left|\theta-\theta^{\prime}\right|<\epsilon} \mathrm{E}_{\theta^{\prime}}\left[L\left(\sqrt{T}\left(\widehat{\theta}_{T}-\theta^{\prime}\right)\right)\right]=\frac{1}{\sqrt{2 \pi}} \int_{\mathbb{R}} L\left(y I(\theta)^{-1 / 2}\right) e^{-y^{2} / 2} d y .
$$


Then for a loss function $L(\cdot) \in \mathcal{L}$ whose growth as $|u| \rightarrow \infty$ is lower than any one of the functions $e^{\epsilon|u|}, \epsilon>0$, we readily deduce that

$$
\lim _{b \rightarrow \infty} \lim _{\epsilon \rightarrow 0} \lim _{T \rightarrow \infty} \sup _{\left|\theta-\theta^{\prime}\right|<\epsilon} \mathrm{E}_{\theta^{\prime}}\left[b \wedge L\left(\sqrt{T}\left(\widehat{\theta}_{T}-\theta^{\prime}\right)\right)\right]=\frac{1}{\sqrt{2 \pi}} \int_{\mathbb{R}} L\left(y I(\theta)^{-1 / 2}\right) e^{-y^{2} / 2} d y .
$$

Thus for the quadratic risk with $L(u)=u^{2}$, we obtain

$$
\lim _{b \rightarrow \infty} \lim _{\epsilon \rightarrow 0} \lim _{T \rightarrow \infty} \sup _{\left|\theta-\theta^{\prime}\right|<\epsilon} \mathrm{E}_{\theta^{\prime}}\left[b \wedge\left(T\left(\widehat{\theta}_{T}-\theta^{\prime}\right)^{2}\right)\right]=I(\theta)^{-1} .
$$

\subsection{Case $F(P)=0, f(\cdot)$ non identically null}

When $F(P)=0$, in Lemma 2 we state that

$$
\lim _{T \rightarrow \infty} \mathcal{L}_{\theta}\left[\left(\frac{J_{T}}{T^{2}}, \frac{U_{T}}{T}\right)\right]=\mathcal{L}\left[\left(\mathcal{I}(\theta), \mathcal{I}(\theta)^{1 / 2} \zeta\right)\right]
$$

in $\mathbb{R}^{2}$ uniformly with respect to $\theta$ varying in any compact subset of $\mathbb{R}$. Here the random Fisher information is defined by $\mathcal{I}(\theta):=\frac{G_{\theta}(P) H_{\theta}(P)}{P^{2}} \int_{0}^{1} \mathrm{~B}_{u}^{2} d u$, and the real-valued random $\zeta$ is Gaussian $\mathcal{N}(0,1)$ independent with respect to the Brownian motion $\left\{\mathrm{B}_{t}: t \in[0,1]\right\}$, so independent with respect to $\mathcal{I}(\theta)$. Then the log-likelihood ratio $\Lambda_{T}^{(\theta, u)}$ can be expressed as

$$
\Lambda_{T}^{(\theta, u)}=u \Delta_{T}^{\theta}-\frac{1}{2} u^{2} \mathcal{I}(\theta)+r_{T}(\theta, u)
$$

where $\Delta_{T}^{\theta}:=T^{-1} U_{T}$ converges in law to the mixed Gaussian law of $\mathcal{I}(\theta)^{1 / 2} \zeta$, and $r_{T}(\theta, u)$ converges in probability to 0 as $T$ goes to infinity. We deduce that the family of laws $\left\{\mathrm{P}_{\theta}^{T}\right.$ : $\theta F(P)>0\}, T>0$, has a likelihood ratio which is LAMN (locally asymptotically mixing normal) (see e.g. Davies 1985; Jeganathan 1982, Definition 3). Following (Jeganathan 1995, Theorem 8; Le Cam and Yang 1990, Section 5.6), for each family $\left\{\bar{\theta}_{T}\right\}$ of estimators of $\theta$ the local minimax risk $R_{\theta}(\bar{\theta})$ can be bounded asymptotically as follows

$$
\lim _{M \rightarrow \infty} \liminf _{T \rightarrow \infty} \sup _{\left|\theta-\theta^{\prime}\right|<M T^{-1}} \mathrm{E}_{\theta^{\prime}}\left[L\left(T\left(\bar{\theta}_{T}-\theta^{\prime}\right)\right)\right] \geq \mathrm{E}\left[L\left(\frac{P}{\sqrt{G_{\theta}(P) H_{\theta}(P)}} \frac{\zeta}{\sqrt{\int_{0}^{1} \mathrm{~B}_{u}^{2} d u}}\right)\right]
$$

for any $L \in \mathcal{L}$. Thanks to Theorem 3 and to (Ibragimov and Has'minskiǐ 1981, Appendix I Theorem 8), we can assert that

Theorem 7 Assume that $F(P)=0$, and $f(\cdot)$ is not identically null. Then the $M L E \widehat{\theta}_{T}$ is locally asymptotically minimax with local scale $T^{-1}$ at any $\theta$ and for any bounded loss function $L(\cdot) \in \mathcal{L}$, in the sense that

$$
\lim _{M \rightarrow \infty} \lim _{T \rightarrow \infty} \sup _{\left|\theta-\theta^{\prime}\right|<M T^{-1}} \mathrm{E}_{\theta^{\prime}}\left[L\left(T\left(\widehat{\theta}_{T}-\theta^{\prime}\right)\right)\right]=\mathrm{E}\left[L\left(\frac{P}{\sqrt{G_{\theta}(P) H_{\theta}(P)}} \frac{\zeta}{\sqrt{\int_{0}^{1} \mathrm{~B}_{u}^{2} d u}}\right)\right] .
$$


Notice that in relation (17) and in Theorem 7, the small neighbourhood $\mathcal{U}_{\theta}$ of $\theta$ in the expression (14) of the local asymptotic minimax risk, is $\left\{\theta^{\prime}:\left|\theta^{\prime}-\theta\right| \leq M T^{-1}\right\}$. Its size is proportional to $T^{-1}$ and tends to 0 as $T \rightarrow \infty$. However when the loss function is quadratic $\left(L(x)=x^{2}\right)$, thanks to van Trees inequality (Gill and Levit 1995) we state the following local asymptotic optimality of the MLE $\widehat{\theta}_{T}$ with small neighbourhood $\mathcal{U}_{\theta}$ with size $\epsilon>0$ as in the LAN case.

Theorem 8 Assume that $F(P)=0, f(\cdot)$ is not identically null. Then for each $\theta \in \mathbb{R}$

$$
\lim _{\epsilon \rightarrow 0} \liminf _{T \rightarrow \infty} \sup _{\left|\theta^{\prime}-\theta\right|<\epsilon} \mathrm{E}_{\theta^{\prime}}\left[T^{2}\left(\bar{\theta}_{T}-\theta^{\prime}\right)^{2}\right] \geq \frac{2 P^{2}}{G_{\theta}(P) H_{\theta}(P)}
$$

where $\left\{\bar{\theta}_{T}\right\}$ is any family of estimators of $\theta$. Furthermore the $M L E \widehat{\theta}_{T}$ is asymptotically minimax for the quadratic loss function in the sense that

$$
\lim _{b \rightarrow \infty} \lim _{\epsilon \rightarrow 0} \lim _{T \rightarrow \infty} \sup _{\left|\theta^{\prime}-\theta\right|<\epsilon} \mathrm{E}_{\theta^{\prime}}\left[b \wedge T^{2}\left(\widehat{\theta}_{T}-\theta^{\prime}\right)^{2}\right]=\frac{2 P^{2}}{G_{\theta}(P) H_{\theta}(P)}
$$

\subsection{Case $\theta F(P)>0$}

From Lemma 3 and the proof of Theorem 5, we know that

$$
\begin{aligned}
& \mathcal{I}^{(\theta)}(t):=\lim _{n \rightarrow \infty} e^{-2 n \theta F(P)} J_{n P+t}=K_{\theta}(t)^{2}\left(\xi_{0}+\zeta_{3}^{(\theta)}\right)^{2} \text { in } \mathrm{L}^{1}\left(\mathrm{P}_{\theta}\right) \text { and } \\
& \lim _{n \rightarrow \infty} \mathcal{L}_{\theta}\left[\left(e^{-2 n \theta F(P)} J_{n P+t}, e^{-n \theta F(P)} U_{n P+t}\right)\right]=\mathcal{L}\left[\mathcal{I}^{(\theta)}(t), \mathcal{I}^{(\theta)}(t)^{1 / 2} \zeta\right] \text { in } \mathbb{R}^{2}
\end{aligned}
$$

the limits being uniform with respect to $t \in[0, P]$ and to $\theta$ varying in any compact subset of $\{\theta: \theta F(P)>0\}$. The random variable $\zeta$ is Gaussian $\mathcal{N}(0,1)$ and independent with respect to $\left\{\mathcal{I}^{(\theta)}(t): t \in[0, P]\right\}$. Thus taking the local scale $\delta_{n P+t}(\theta):=e^{-n \theta F(P)}$, from equality (15) we obtain that

$$
\Lambda_{n P+t}^{(\theta, u)}=u \Delta_{n P+t}^{(\theta)}-\frac{1}{2} u^{2} \mathcal{I}^{(\theta)}(t)+r_{n P+t}(\theta, u)
$$

where for any $t \in[0, P]$, the law of $\Delta_{n P+t}^{(\theta)}:=e^{-n \theta F(P)} U_{n P+t}$ converges to the mixed law of $\mathcal{I}^{(\theta)}(t)^{1 / 2} \zeta$, and $r_{n P+t}(\theta, u)$ converges in probability to 0 as $n$ goes to infinity. Notice that the local scale depends on $\theta$.

We deduce that for each $t \in[0, P]$, the sequence of families of laws $\left\{\mathrm{P}_{\theta}^{n P+t}: \theta F(P)>0\right\}$, $n \geq 1$, has a likelihood ratio which is LAMN. Then for each sequence $\left\{\bar{\theta}_{n P+t}\right\}$ of estimators of $\theta$, the local asymptotic minimax risk (14) can be bounded as follows.

$$
\lim _{M \rightarrow \infty} \liminf _{n \rightarrow \infty} \sup _{\left|\theta-\theta^{\prime}\right|<M e^{-n \theta F(P)}} \mathrm{E}_{\theta^{\prime}}\left[L\left(e^{n \theta^{\prime} F(P)}\left(\bar{\theta}_{n P+t}-\theta^{\prime}\right)\right)\right] \geq \mathrm{E}\left[L\left(\frac{\zeta}{K_{\theta}(t)\left(\xi_{0}+\zeta_{3}^{(\theta)}\right)}\right)\right]
$$

for any $t \in[0, P]$ and any $L \in \mathcal{L}$, each estimator $\bar{\theta}_{n P+t}$ being measurable with respect the observation $\left\{\xi_{s}: s \in[0, n P+t]\right\}$. Thanks to Theorem 5 we can assert the following optimality of the MLE. 
Theorem 9 Assume that $F(P) \neq 0$. Then for each $t \in[0, P]$, the $M L E \widehat{\theta}_{n P+t}$ is locally asymptotically minimax with local scale $e^{-n \theta F(P)}$ at any $\theta$ such $\theta F(P)>0$, and for any bounded loss function $L(\cdot) \in \mathcal{L}$, in the sense that

$$
\lim _{M \rightarrow \infty} \lim _{n \rightarrow \infty} \sup _{\left|\theta-\theta^{\prime}\right|<M e^{-n \theta F(P)}} \mathrm{E}_{\theta^{\prime}}\left[L\left(e^{n \theta^{\prime} F(P)}\left(\widehat{\theta}_{n P+t}-\theta^{\prime}\right)\right)\right]=\mathrm{E}\left[L\left(\frac{\zeta}{K_{\theta}(t)\left(\xi_{0}+\zeta_{3}^{(\theta)}\right)}\right)\right] .
$$

The previous minimax result in the case $\theta F(P)>0$ concerns the subsequences $\left\{\widehat{\theta}_{n P+t}\right\}_{n}$, $t \in[0, P]$, of the family of MLE $\left\{\widehat{\theta}_{T}, T>0\right\}$ of $\theta$. We can deduce the local asymptotic minimax optimality for the whole family $\left\{\widehat{\theta}_{T}, T>0\right\}$ as follows.

Let $\delta_{T}(\theta):=\left(K_{\theta}(t) e^{n \theta F(P)}\right)^{-1}$ where $T=n P+t$ with $n \in \mathbb{N}$ and $t \in[0, P)$. From the lower bound inequality (19) as well as the fact that the function $(\theta, t) \mapsto K_{\theta}(t)$ is positive continuous on $\{\theta: \theta F(P)>0\} \times[0, P)$, we obtain that

$$
\lim _{M \rightarrow \infty} \liminf _{T \rightarrow \infty} \sup _{\left|\theta-\theta^{\prime}\right|<M \delta_{T}(\theta)} \mathrm{E}_{\theta^{\prime}}\left[L\left(\delta_{T}\left(\theta^{\prime}\right)^{-1}\left(\bar{\theta}_{T}-\theta^{\prime}\right)\right)\right] \geq \mathrm{E}\left[L\left(\frac{\zeta}{\xi_{0}+\zeta_{3}^{(\theta)}}\right)\right]
$$

for any $\theta \in \mathbb{R}$ such $\theta F(P)>0$, any bounded loss function $L(\cdot) \in \mathcal{L}$ and any family $\left\{\bar{\theta}_{T}\right\}$ of estimators of $\theta$. Then Theorem 9 implies that the MLE $\widehat{\theta}_{T}$ is optimal in the sense that

$$
\lim _{M \rightarrow \infty} \lim _{T \rightarrow \infty} \sup _{\left|\theta-\theta^{\prime}\right|<M \delta_{T}(\theta)} \mathrm{E}_{\theta^{\prime}}\left[L\left(\delta_{T}\left(\theta^{\prime}\right)^{-1}\left(\widehat{\theta}_{T}-\theta^{\prime}\right)\right)\right]=\mathrm{E}\left[L\left(\frac{\zeta}{\xi_{0}+\zeta_{3}^{(\theta)}}\right)\right] .
$$

Remark that the limit law in relation (13) is not square-integrable. Hence when $\theta F(P)>0$, we cannot get a finite local asymptotic minimax lower bound for the quadratic risk with small neighbourhood $\mathcal{U}_{\theta}$ of type $\left\{\theta^{\prime}:\left|\theta^{\prime}-\theta\right| \leq M e^{-n \theta F(P)}\right\}$.

\section{Proofs}

\subsection{Proofs of results in Section 3}

\subsubsection{Proof of Theorem 1}

From the remarks before the statement of Theorem 1 it is only sufficient to prove that $J_{\infty}=\infty$ a.e.. Recall that the function $f(\cdot)$ is not identically null and notice that the quadratic variation $J_{T}$ increases to $J_{\infty}$ a.e. as $T \rightarrow \infty$.

(i) First assume that $\theta F(P)<0$. From Section 2 we know that in this case the $P$-segments Markov sequence $\left\{\mathbf{X}_{k}\right\}$ is positive Harris recurrent. Then the ergodic theorem (Höpfner and Kutoyants 2010, Theorem 2.1) and limit (3) entail that

$$
\lim _{T \rightarrow \infty} \frac{J_{T}}{T}=\lim _{T \rightarrow \infty} \frac{1}{T} \int_{0}^{T} f(u)^{2} \xi_{u}^{2} d u=I(\theta)>0 \quad \mathrm{P}_{\theta} \text {-a.e. }
$$

where $I(\theta)$ is defined in relation (12). This implies that $J_{\infty}=\infty$ a.e. 
(ii) Assume now that $\theta F(P)=0$. For any $n>0$ we have

$$
J_{n P} \geq \int_{0}^{P} f(u)^{2} \xi_{n P+u}^{2} d u
$$

In another hand limit (4) implies that

$$
\lim _{n \rightarrow \infty} \mathrm{P}_{\theta}\left[\frac{1}{n} \int_{0}^{P} f(u)^{2} \xi_{n P+u}^{2} d u \geq a\right]=\mathrm{P}\left[H_{\theta}(P)\left(\zeta_{2}^{(\theta)}\right)^{2} \geq a\right]
$$

for any $a>0$, and where $\mathcal{L}\left(\zeta_{2}^{(\theta)}\right)=\mathcal{N}\left(0, G_{\theta}(P)\right)$. As the function $f(\cdot)$ is not identically null, we have $G_{\theta}(P) H_{\theta}(P)>0$ so the random variable $\zeta_{2}^{(\theta)}$ is non-degenerate Gaussian and we readily obtain that $J_{n P}$ converges in $\mathrm{P}_{\theta}$-probability to infinity as $n \rightarrow \infty$. As $J_{n P}$ increases to $J_{\infty}$, $\mathrm{P}_{\theta}$-a.e. as $T \rightarrow \infty$, we deduce that $\mathrm{P}_{\theta}\left[J_{\infty}<\infty\right]=0$.

(iii) Finally assume that $\theta F(P)>0$. According to limit (5)

$$
\lim _{n \rightarrow \infty} e^{-2 n \theta F(P)} \int_{0}^{\cdot} f(u)^{2} \xi_{n P+u}^{2} d u=H_{\theta}(\cdot)\left(\xi_{0}+\zeta_{3}^{(\theta)}\right)^{2} \quad \mathrm{P}_{\theta^{-}} \text {a.e. in } \mathcal{C}[0, P]
$$

where $\zeta_{3}^{(\theta)}$ is the sum of the P-a.e. convergent series $\sum_{k} e^{-k \theta F(P)} \mathbf{Z}_{k}(P)$. Thus $\mathcal{L}\left(\zeta_{3}^{(\theta)}\right)=$ $\mathcal{N}\left(0, \frac{G_{\theta}(P}{1-e^{-2 \theta F(P)}}\right)$ and the real-valued random variables $\xi_{0}$ and $\zeta_{3}^{(\theta)}$ are independent. Besides $J_{n P}$ can be expressed as

$$
J_{n P}=\sum_{k=0}^{n-1} \int_{0}^{P} f(u)^{2} \xi_{k P+u}^{2} d u=\sum_{k=0}^{n-1} e^{2 k \theta F(P)} e^{-2 k \theta F(P)} \int_{0}^{P} f(u)^{2} \xi_{k P+u}^{2} d u
$$

for any positive integer $n$. Applying Toeplitz lemma on series convergence we obtain

$$
\lim _{n \rightarrow \infty} e^{-2 n \theta F(P)} J_{n P}=\frac{H_{\theta}(P)}{e^{2 \theta F(P)}-1}\left(\xi_{0}+\zeta_{3}^{(\theta)}\right)^{2} \quad \mathrm{P}_{\theta^{-}} \text {a.e. in } \mathbb{R} .
$$

Since $\xi_{0}$ and $\zeta_{3}^{(\theta)}$ are independent and $\zeta_{3}^{(\theta)}$ is non-degenerate Gaussian, we have $\mathrm{P}_{\theta}\left[\xi_{0}+\zeta_{3}^{(\theta)}=0\right]=$ 0 . Hence $J_{\infty}=\infty \mathrm{P}_{\theta}$-a.e. This completes the proof of the theorem.

\subsubsection{Proof of Theorem 2}

Recall that $J_{T}\left(\widehat{\theta}_{T}-\theta\right)=U_{T}$ where $\left\{J_{t}, t \geq 0\right\}$ is the quadratic variation process of the martingale $\left\{U_{t}, t \geq 0\right\}$ defined by relation (10). The recurrence property of the process $\left\{\xi_{t}, t>\right.$ $0\}$, implies that $J_{T}$ converges almost surely to $I(\theta)$ as $T \rightarrow \infty$. Then the central limit theorem for integrales with respect to Brownian motion (see e.g. Feigin 1976; see also Kutoyants 2004, Theorem 1.19; Barzcy and Pap 2010) entails the convergence in law

$$
\lim _{T \rightarrow \infty} \mathcal{L}_{\theta}\left[\sqrt{J_{T}}\left(\widehat{\theta}_{T}-\theta\right)\right]=\mathcal{N}(0,1)
$$

for any $\theta$ such that $\theta F(P)<0$. To establish the uniform convergence with respect to $\theta$, requires a little more computations concerning the asymptotic behaviour of $J_{T} / T$. 
Let $n \in \mathbb{N}$ and $t \in[0, P]$. Then

$$
J_{n P+t}=\int_{0}^{n P+t} f(u)^{2} \xi_{u}^{2} d u=\sum_{k=0}^{n-1} \int_{0}^{P} f(u)^{2} \xi_{k P+u}^{2} d u+\int_{0}^{t} f(u)^{2} \xi_{n P+u}^{2} d u
$$

Owing to expression (6) we readily obtain by induction the following decomposition of $\xi_{n P+u}$

$$
\xi_{k P+u}=e^{\theta F(u)}\left(e^{k \theta F(P)}\left(\xi_{0}+S_{k-1}\right)+\mathbf{Z}_{k}(u)\right)
$$

where

$$
S_{-1}:=0 \quad \text { and } \quad S_{k-1}:=\sum_{j=0}^{k-1} e^{-j \theta F(P)} Z_{j}=\int_{0}^{k P} e^{-\theta F(v)} d \mathrm{~B}_{v}
$$

for any $k \geq 1$, and $Z_{j}:=\mathbf{Z}_{j}(P)$ for $j \in \mathbb{N}$. Thus we can write $J_{n P+t}$ as

$$
\begin{aligned}
J_{n P+t}= & H_{\theta}(P) \sum_{k=0}^{n-1} e^{2 k \theta F(P)}\left(\xi_{0}+S_{k-1}\right)^{2}+\int_{0}^{P} f(u)^{2} e^{2 \theta F(u)} \sum_{k=0}^{n-1} \mathbf{Z}_{k}(u)^{2} d u \\
& +2 \sum_{k=0}^{n-1} e^{k \theta F(P)}\left(\xi_{0}+S_{k-1}\right) \int_{0}^{P} f(u)^{2} e^{2 \theta F(u)} \mathbf{Z}_{k}(u) d u+H_{\theta}(t) e^{2 n \theta F(P)}\left(\xi_{0}+S_{n-1}\right)^{2} \\
& +\int_{0}^{t} f(u)^{2} e^{2 \theta F(u)} \mathbf{Z}_{n}(u)^{2} d u+2 e^{n \theta F(P)}\left(\xi_{0}+S_{n-1}\right) \int_{0}^{t} f(u)^{2} e^{2 \theta F(u)} \mathbf{Z}_{n}(u) d u \\
:= & A_{1}+A_{2}+A_{3}+A_{4}+A_{5}+A_{6} .
\end{aligned}
$$

Using the facts that the processes $\mathbf{Z}_{n}$ s are independent, and each of them is zero-mean Gaussian with independent increments, $\operatorname{var}_{\theta}\left[\mathbf{Z}_{k}(u)\right]=G_{\theta}(u)$ for $u \in[0, P]$, we will establish in the following Lemma 1 that $\left(A_{1}+A_{2}\right) / n$ converges in $\mathrm{P}_{\theta}$-quadratic mean to $P I(\theta)$, and $A_{3} / n$, converges to 0 . With the same argument we can prove that $A_{4} / n, A_{5} / n$ and $A_{6} / n$ converge to 0 . Each convergence being uniform with respect to $t \in[0, P]$ and to $\theta$ in any compact subset of $\{\theta \in \mathbb{R}: \theta F(P)<0\}$.

For each interger $n$, the $P$-segments process $\mathbf{Z}_{n}=\left\{\mathbf{Z}_{n}(t), t \in[0, P]\right\}$ is a continuous martingale with respect to the filtration generated by the Brownian motion $\mathrm{B}^{(n P)}=\left\{\mathrm{B}_{n P+t}-\mathrm{B}_{n P}, t \in\right.$ $[0, P]\}$, and Doob maximal equality for matingales entails that

$$
\mathrm{E}_{\theta}\left[\max _{t \in[0, P]} \mathbf{Z}_{n}(t)^{2}\right] \leq 2 \int_{0}^{P} e^{-2 \theta F(u)} d u=2 G_{\theta}(u) .
$$

Thus we obtain that

$$
\lim _{T \rightarrow \infty} \frac{J_{T}}{T}=I(\theta)
$$

in $\mathrm{P}_{\theta}$-quadratic mean uniformly with respect to $\theta$ varying in any compact subset of $\{\theta: \theta F(P)<$ $0\}$. Thanks to the uniform central limit theorem for integrales with respect to Brownian motion (Kutoyants 2004, Theorem 1.20) we deduce the assertions of the theorem.

To complete the proof of Theorem 2 we state the convergence of $A_{1} / n, A_{2} / n$ and $A_{3} / n$. 
Lemma 1 Uniformly with respect to $t \in[0, P]$ and to $\theta$ in any compact subset of $\{\theta \in \mathbb{R}$ : $\theta F(P)<0\}$, we have

$$
\begin{gathered}
\lim _{n \rightarrow \infty} \mathrm{E}_{\theta}\left[\left(\frac{A_{1}}{n}-\frac{G_{\theta}(P) H_{\theta}(P)}{e^{-2 \theta F(P)}-1}\right)^{2}\right]=0, \\
\lim _{n \rightarrow \infty} \mathrm{E}_{\theta}\left[\left(\frac{A_{2}}{n}-\int_{0}^{P} f(u)^{2} e^{2 \theta F(u)} G_{\theta}(u) d u\right)^{2}\right]=0 .
\end{gathered}
$$

and

$$
\lim _{n \rightarrow \infty} \frac{\mathrm{E}_{\theta}\left[A_{3}^{2}\right]}{n^{2}}=0
$$

Proof Let $K_{-}$be any compact subset contained in $\{\theta: \theta F(P)<0\}$.

(i) Term $A_{1}$. First express $A_{1}$ as

$$
A_{1}=H_{\theta}(P) \sum_{k=0}^{n-1} e^{2 k \theta F(P)} \xi_{0}^{2}+H_{\theta}(P) \sum_{k=0}^{n-1} e^{2 k \theta F(P)} S_{k-1}^{2}+2 H_{\theta}(P) \sum_{k=0}^{n-1} e^{2 k \theta F(P)} \xi_{0} S_{k-1} .
$$

Since $\theta F(P)<0$, the first term of expression (26) converges to $H_{\theta}(P) \xi_{0}^{2} /\left(1-e^{2 \theta F(P)}\right)$ uniformly with respect to $\theta \in K_{-}$.

Next we have

$$
\sum_{k=0}^{n-1} e^{2 k \theta F(P)} S_{k-1}^{2}=\sum_{j=0}^{n-2} a_{j} Z_{j}^{2}+2 \sum_{j=0}^{n-3} \sum_{j^{\prime}=j+1}^{n-2} b_{j j^{\prime}} Z_{j} Z_{j^{\prime}}
$$

where

$$
a_{j}=\frac{e^{2 \theta F(P)}-e^{2(n-j) \theta F(P)}}{1-e^{2 \theta F(P)}} \quad \text { and } \quad b_{j j^{\prime}}=\frac{e^{-j \theta F(P)}\left(e^{\left(2+j^{\prime}\right) \theta F(P)}-e^{\left(2 n-j^{\prime}\right) \theta F(P)}\right)}{1-e^{2 \theta F(P)}} .
$$

Thanks to their definition, the random variables $Z_{j}$ s are independent and have the same Gaussian law $\mathcal{N}\left(0, G_{\theta}(P)\right)$. Thus we obtain that

$$
\mathrm{E}_{\theta}\left[\sum_{k=0}^{n-1} e^{2 k \theta F(P)} S_{k-1}^{2}\right]=\sum_{j=0}^{n-2} a_{j} G_{\theta}(P)=\frac{1}{1-e^{2 \theta F(P)}}\left((n-1) e^{2 \theta F(P)}-\frac{e^{2 n \theta F(p)}-1}{1-e^{-2 \theta F(P)}}\right) G_{\theta}(P)
$$

and, owing to the expression of the fouth moment of a Gaussian vector in terms of its second moments and first moments (Isserli's formula),

$$
\begin{aligned}
\operatorname{var}_{\theta}\left[\sum_{k=0}^{n-1} e^{2 k \theta F(P)} S_{k-1}^{2}\right] & \leq 2\left(\sum_{j=0}^{n-2} a_{j}^{2} G_{\theta}(P)^{2}+4 \sum_{j=0}^{n-3} \sum_{j^{\prime}=j+1}^{n-2} b_{j j^{\prime}}^{2} G_{\theta}(P)^{2}\right) \\
& \leq \frac{4 n G_{\theta}(P)^{2}}{\left(1-e^{2 \theta F(P)}\right)^{2}}+\frac{32 n G_{\theta}(P)^{2}}{\left(1-e^{2 \theta F(P)}\right)^{2}}\left(\frac{1}{1-e^{2 \theta F(P)}}+\frac{1}{e^{-2 \theta F(P)}-1}\right) .
\end{aligned}
$$

We deduce that

$$
\lim _{n \rightarrow \infty} \mathrm{E}_{\theta}\left[\left(\frac{1}{n} \sum_{k=0}^{n-1} e^{2 k \theta F(P)} S_{k-1}^{2}-\frac{G_{\theta}(P)}{e^{-2 \theta F(P)}-1}\right)^{2}\right]=0
$$


uniformly with respect to $\theta$ in the compact subset $K_{-}$.

As for the third term of $A_{1}$ we have

$$
\sum_{k=0}^{n-1} e^{2 k \theta F(P)} S_{k-1}=\sum_{j=0}^{n-2}\left(\frac{e^{2(j+1) \theta F(P)}-e^{2 n \theta F(P)}}{1-e^{2 \theta F(P)}}\right) e^{-j \theta F(P)} Z_{j}
$$

which converges to

$$
\frac{e^{2 \theta F(P)}}{1-e^{2 \theta F(P)}} \sum_{j=0}^{\infty} e^{j \theta F(P)} Z_{j}
$$

in quadratic mean uniformly with respect to $\theta$ in the compact subset $K_{-}$. Hence convergence (23) is proved.

(ii) Term $A_{2}$. From the definition of $A_{2}$ and relation (2), we readily have

$$
\mathrm{E}_{\theta}\left[A_{2}\right]=n \int_{0}^{P} f(u)^{2} e^{2 \theta F(u)} G_{\theta}(u) d u .
$$

The independence of the Gaussian processes $\mathbf{Z}_{k}$ and $\mathbf{Z}_{k^{\prime}}$ for $k \neq k^{\prime}$ entails that

$$
\operatorname{var}_{\theta}\left[A_{2}\right]=\sum_{k=0}^{n-1} \int_{0}^{P} \int_{0}^{P} f(u)^{2} f\left(u^{\prime}\right)^{2} e^{2 \theta\left(F(u)+F\left(u^{\prime}\right)\right)} \operatorname{cov}_{\theta}\left[\mathbf{Z}_{k}(u)^{2}, \mathbf{Z}_{k}\left(u^{\prime}\right)^{2}\right] d u d u^{\prime} .
$$

By Isserli's formula, $\operatorname{cov}_{\theta}\left[\mathbf{Z}_{k}(u)^{2}, \mathbf{Z}_{k}\left(u^{\prime}\right)^{2}\right]=2 G_{\theta}(u)^{2}$ for $u \leq u^{\prime}$, we deduce that

$$
\operatorname{var}_{\theta}\left[A_{2}\right]=4 n \int_{0}^{P} \int_{u}^{P} f(u)^{2} f\left(u^{\prime}\right)^{2} e^{2 \theta\left(F(u)+F\left(u^{\prime}\right)\right)} G_{\theta}(u)^{2} d u d u^{\prime} .
$$

Consequently convergence (24) is proved.

(iii) Term $A_{3}$. The independence between the processes $\mathbf{Z}_{k}$ and $\mathbf{Z}_{k^{\prime}}$ for $k \neq k^{\prime}$ implies that $\mathrm{E}_{\theta}\left[A_{3}\right]=0$. Furthermore, since $\xi_{0}$ is independent with respect to the process $\mathbf{Z}_{k}$, and $\operatorname{cov}_{\theta}\left[\mathbf{Z}_{k}(u)^{2}, \mathbf{Z}_{k}\left(u^{\prime}\right)^{2}\right]=2 G_{\theta}(u)^{2}$ for $u \leq u^{\prime}$, we have

$$
\begin{aligned}
\operatorname{var}_{\theta} & {\left[\sum_{k=0}^{n-1} e^{k \theta F(P)} \xi_{0} \int_{0}^{P} f(u)^{2} e^{2 \theta F(u)} \mathbf{Z}_{k}(u) d u\right] } \\
& =2 \mathrm{E}_{\theta}\left[\xi_{0}^{2}\right] \frac{1-e^{2 n \theta F(P)}}{1-e^{2 \theta F(P)}} \int_{0}^{P} \int_{u}^{P} f(u)^{2} f\left(u^{\prime}\right)^{2} e^{2 \theta\left(F(u)+F\left(u^{\prime}\right)\right)} G_{\theta}(u) d u d u^{\prime}
\end{aligned}
$$

and

$$
\begin{aligned}
\operatorname{var}_{\theta} & {\left[\sum_{k=0}^{n-1} e^{k \theta F(P)} S_{k-1} \int_{0}^{P} f(u)^{2} e^{2 \theta F(u)} \mathbf{Z}_{k}(u) d u\right] } \\
& =\sum_{k=1}^{n-1} \sum_{j=0}^{k-1} e^{2(k-j) \theta F(P)} \int_{0}^{P} \int_{0}^{P} f(u)^{2} f\left(u^{\prime}\right)^{2} e^{2 \theta\left(F(u)+F\left(u^{\prime}\right)\right)} \mathrm{E}_{\theta}\left[Z_{j}^{2}\right] \mathrm{E}_{\theta}\left[\mathbf{Z}_{k}(u) \mathbf{Z}_{k}\left(u^{\prime}\right)\right] d u d u^{\prime} \\
& =\frac{2 G_{\theta}(P)}{e^{-2 \theta F(P)}-1}\left(n-\frac{1-e^{2 n \theta F(P)}}{1-e^{2 \theta F(P)}}\right) \int_{0}^{P} \int_{u}^{P} f(u)^{2} f\left(u^{\prime}\right)^{2} e^{2 \theta\left(F(u)+F\left(u^{\prime}\right)\right)} G_{\theta}(u) d u d u^{\prime},
\end{aligned}
$$


the first equality being due to the independence between the Gaussian processes $\mathbf{Z}_{k}, k \in \mathbb{N}$ and Isserli's formula. The second equality is a direct consequence of the definition of $\mathbf{Z}_{k}$. Thus convergence (25) is proved.

\subsubsection{Proof of Theorem 3}

As we do not prove the convergence of $J_{T} / T^{2}$ in probability, the results in (Barczy and Pap 2010) cannot be applied here to establish Theorem 3. Actually this theorem is a direct consequence of the following result.

Lemma 2 Assume that $F(P)=0$ and $f(\cdot)$ not identically null. Then

$$
\lim _{T \rightarrow \infty} \mathcal{L}_{\theta}\left[\left(\frac{J_{T}}{T^{2}}, \frac{U_{T}}{T}\right)\right]=\mathcal{L}\left[\left(\frac{G_{\theta}(P) H_{\theta}(P)}{P^{2}} \int_{0}^{1} \mathrm{~B}_{u}^{2} d u, \frac{\sqrt{G_{\theta}(P) H_{\theta}(P)}}{P} \int_{0}^{1} \mathrm{~B}_{u} d \mathrm{~B}_{u}^{\prime}\right)\right]
$$

uniformly with respect to $\theta$ varying in any compact subset of $\mathbb{R}$, where $\left\{\mathrm{B}_{t}, t \in[0,1]\right\}$ and $\left\{\mathrm{B}_{t}^{\prime}, t \in[0,1]\right\}$ are two independent Brownian motions.

Notice that the random variable

$$
\zeta:=\frac{\int_{0}^{1} \mathrm{~B}_{u} d \mathrm{~B}_{u}^{\prime}}{\sqrt{\int_{0}^{1} \mathrm{~B}_{u}^{2} d u}}
$$

is Gaussian $\mathcal{N}(0,1)$, and is independent with respect to the Brownian motion $\left\{\mathrm{B}_{t}, t \in[0,1]\right\}$.

Proof The proof of relation (27) is based on the method presented by Phillips (1987) who studied the asymptotic approach of an autoregressive time series with a root near the unit by an Ornstein Uhlenbeck time-continuous process. First we establish that the asymptotic behaviours of $J_{n P+t}$ and of $U_{n P+t}$ coincide with those of sums of time series (formulae (29) and (31)). Next we apply Phillips'method to conclude.

(i) $J_{n P+t}$. Let $n \in \mathbb{N}$ and $t \in[0, P]$ and consider decomposition (21) with $F(P)=0$. The random variables $Z_{j}, j=0, \ldots, n$, are independent with the same law $\mathcal{N}\left(0, G_{\theta}(P)\right)$, so thanks to inequality (22), the first term is equal to

$$
A_{1}=H_{\theta}(P) \sum_{k=0}^{n-1} S_{k-1}^{2}+o_{\mathrm{P}_{\theta}}\left(n^{2}\right)
$$

as $n \rightarrow \infty$, and $A_{4}$ is $o_{\mathrm{P}_{\theta}}\left(n^{2}\right)$. Furthermore inequality (22) also entails that $A_{2}$ is $\mathcal{O}_{\mathrm{P}_{\theta}}(n)$, and $A_{5}$ is $\mathcal{O}_{\mathrm{P}_{\theta}}(1)$. With the previous arguments we easily obtain that $A_{3}$ and $A_{6}$ are $o_{\mathrm{P}_{\theta}}(n)$. Hence we deduce that

$$
J_{n P+t}=H_{\theta}(P) \sum_{k=0}^{n-1} S_{k-1}^{2}+o_{\mathrm{P}_{\theta}}\left(n^{2}\right)
$$


as $n \rightarrow \infty$. Here the $\mathcal{O}_{\mathrm{P}_{\theta}}(\cdot) \mathrm{s}$ and $o_{\mathrm{P}_{\theta}}(\cdot) \mathrm{s}$ are uniform with respect to $t \in[0, P]$ and with respect to $\theta$ varying in any compact subset of $\mathbb{R}$.

(ii) $U_{n P+t}$. From decomposition (20) we can express $U_{n P+t}$ as

$$
U_{n P+t}=\sum_{k=0}^{n-1}\left(Y_{k}\left(\xi_{0}+S_{k-1}\right)+W_{k}\right)+\mathbf{Y}_{n}(t)\left(\xi_{0}+S_{n-1}\right)+\mathbf{W}_{n}(t)
$$

where $Y_{k}:=\mathbf{Y}_{k}(P), W_{k}:=\mathbf{W}_{k}(P)$,

$$
\begin{aligned}
\mathbf{Y}_{k}(t) & :=\int_{0}^{t} f(u) e^{\theta F(u)} d \mathrm{~B}_{u}^{(k P)} \text { and } \\
\mathbf{W}_{k}(t) & :=\int_{0}^{t} f(u) e^{\theta F(u)} \mathbf{Z}_{k}(u) d \mathrm{~B}_{u}^{(k P)}=\int_{0}^{t}\left(\int_{0}^{u} f(u) e^{\theta(F(u)-F(v))} d \mathrm{~B}_{v}^{(k P)}\right) d \mathrm{~B}_{u}^{(k P)}
\end{aligned}
$$

for any $k \in \mathbb{N}$ and any $t \in[0, P]$. The random variables $Y_{k} \mathrm{~s}$, are independent with the same law $\mathcal{N}\left(0, H_{\theta}(P)\right)$. The random variables $W_{k} \mathrm{~s}$ are also independent with the same law which is with zero-mean and finite variance. Then the law of large numbers entails that $U_{n P}$, the first term of expression (30), is equal to

$$
\sum_{k=0}^{n-1} Y_{k} S_{k-1}+o_{\mathrm{P}_{\theta}}(n)
$$

as $n \rightarrow \infty$ uniformly with respect to $\theta$ varying in any compact subset of $\mathbb{R}$. For each integer $n$, the processes $\left\{\mathbf{Y}_{n}(t), t \in[0, P]\right\}$ and $\left\{\mathbf{W}_{n}(t), t \in[0, P]\right\}$ are continuous martingales with respect to the filtration $\left\{\mathcal{F}_{t}^{(n P)}, t \in[0, P]\right\}$ generated by the Brownian motion $\left\{\mathrm{B}_{t}^{(n P)}, t \in[0, P]\right\}$. Owing to Doob maxima inequality for continuous martingales we have

$$
\begin{aligned}
& \mathrm{E}_{\theta}\left[\max _{t \in[0, P]} \mathbf{Y}_{n}(t)^{2}\right] \leq 4 \mathrm{E}_{\theta}\left[\mathbf{Y}_{n}(P)^{2}\right]=4 H_{\theta}(P)<\infty \\
& \mathrm{E}_{\theta}\left[\max _{t \in[0, P]} \mathbf{W}_{n}(t)^{2}\right] \leq 4 \mathrm{E}_{\theta}\left[\mathbf{W}_{n}(P)^{2}\right]=4 \int_{0}^{P} f(u)^{2} e^{2 \theta F(u)} G_{\theta}(u) d u<\infty .
\end{aligned}
$$

Since $\mathrm{E}_{\theta}\left[S_{n}^{2}\right]$ is $o\left(n^{2}\right)$, we deduce that the second term of expression (30) is of order $o_{\mathrm{P}_{\theta}}(n)$ and the third term is $\mathcal{O}_{\mathrm{P}_{\theta}}(1)$, as $n \rightarrow \infty$. Hence

$$
U_{n P+t}=\sum_{k=0}^{n-1} Y_{k} S_{k-1}+o_{\mathrm{P}_{\theta}}(n)
$$

uniformly with respect to $t \in[0, P]$ and to $\theta$ varying in any compact subset of $\mathbb{R}$ as $n \rightarrow \infty$.

(iii) Thanks to the previous computations, it remains to study the asymptotic behaviour of the random vector

$$
\left(\frac{1}{n^{2}} \sum_{k=1}^{n-1} S_{k-1}^{2}, \frac{1}{n} \sum_{k=1}^{n-1} Y_{k} S_{k-1}\right)=\left(\frac{1}{n^{2}} \sum_{k=1}^{n-1}\left(\sum_{j=0}^{k-1} Z_{j}\right)^{2}, \frac{1}{n} \sum_{k=1}^{n-1} \sum_{j=0}^{k-1} Y_{k} Z_{j}\right) .
$$


For that we are going to construct another random vector with the same law and which converges in probability as $n \rightarrow \infty$ to the required limit (see e.g. Phillips 1987). Since

$$
Y_{j}=\int_{j P}^{(j+1) P} f(u) e^{\theta F(u)} d \mathrm{~B}_{u}, \quad \text { and } \quad Z_{j}=\int_{j P}^{(j+1) P} e^{-\theta F(u)} d \mathrm{~B}_{u}
$$

for any $j \in \mathbb{N}$, the random vector $\left(Y_{0}, \ldots, Y_{n-1}, Z_{0}, \ldots, Z_{n-1}\right)$ is Gaussian and $\mathrm{E}_{\theta}\left[Y_{j} Y_{k}\right]=$ $\mathrm{E}_{\theta}\left[Y_{j} Z_{k}\right]=\mathrm{E}_{\theta}\left[Z_{j} Z_{k}\right]=0$, for all integers $j \neq k$. Furthermore

$$
\mathrm{E}_{\theta}\left[Y_{j} Z_{j}\right]=\int_{j P}^{(j+1) P} f(u) e^{\theta F(u)} \times e^{-\theta F(u)} d u=\int_{j P}^{(j+1) P} f(u) d u=F(P)=0
$$

for any integer $j$. Owing to the properties of Gaussian vectors, we deduce that the random variables $Y_{0}, \ldots, Y_{n-1}, Z_{0}, \ldots, Z_{n-1}$ are independent, $\mathcal{L}_{\theta}\left(Y_{j}\right)=\mathcal{N}\left(0, H_{\theta}(P)\right)$ and $\mathcal{L}_{\theta}\left(Z_{j}\right)=$ $\mathcal{N}\left(0, G_{\theta}(P)\right)$ for any integer $j$. Now consider $\left\{\mathrm{B}_{t}, t \in[0,1]\right\}$ and $\left\{\mathrm{B}_{t}^{\prime}, t \in[0,1]\right\}$ two independent Brownian motions and let

$$
Y_{j}^{(\theta)}:=\sqrt{n H_{\theta}(P)}\left(\mathrm{B}_{\frac{j+1}{n}}^{\prime}-\mathrm{B}_{\frac{j}{n}}^{\prime}\right) \quad \text { and } \quad Z_{j}^{(\theta)}:=\sqrt{n G_{\theta}(P)}\left(\mathrm{B}_{\frac{j+1}{n}}-\mathrm{B}_{\frac{j}{n}}\right)
$$

for any $j=0, \ldots, n-1$. Then the random variables $Y_{0}^{(\theta)}, \ldots, Y_{n-1}^{(\theta)}, Z_{0}^{(\theta)}, \ldots, Z_{n-1}^{(\theta)}$ are independent and $\mathcal{L}_{\theta}\left(Y_{0}, \ldots, Y_{n-1}, Z_{0}, \ldots, Z_{n-1}\right)=\mathcal{L}\left(Y_{0}^{(\theta)}, \ldots, Y_{n-1}^{(\theta)}, Z_{0}^{(\theta)}, \ldots, Z_{n-1}^{(\theta)}\right)$. As a consequence,

$\mathcal{L}_{\theta}\left[\left(\frac{1}{n^{2}} \sum_{k=1}^{n-1}\left(\sum_{j=0}^{k-1} Z_{j}\right)^{2}, \frac{1}{n} \sum_{k=1}^{n-1} \sum_{j=0}^{k-1} Y_{k} Z_{j}\right)\right)=\mathcal{L}\left[\left(\frac{1}{n^{2}} \sum_{k=1}^{n-1}\left(\sum_{j=0}^{k-1} Z_{j}^{(\theta)}\right)^{2}, \frac{1}{n} \sum_{k=1}^{n-1} \sum_{j=0}^{k-1} Y_{k}^{(\theta)} Z_{j}^{(\theta)}\right)\right]$.

Furthermore

$$
\frac{1}{n^{2}} \sum_{k=1}^{n-1}\left(\sum_{j=0}^{k-1} Z_{j}^{(\theta)}\right)^{2}=\frac{G_{\theta}(P)}{n} \sum_{k=1}^{n-1}\left(\mathrm{~B}_{\frac{k}{n}}\right)^{2}
$$

and

$$
\frac{1}{n} \sum_{k=1}^{n-1} \sum_{j=0}^{k-1} Y_{k}^{(\theta)} Z_{j}^{(\theta)}=\sqrt{G_{\theta}(P) H_{\theta}(P)} \sum_{k=1}^{n-1}\left(\mathrm{~B}_{\frac{k+1}{n}}^{\prime}-\mathrm{B}_{\frac{k}{n}}^{\prime}\right) \mathrm{B}_{\frac{k}{n}} .
$$

We know that any Brownian motion admits a version such that P-almost all its sample paths are continuous. For such a version of $\left\{\mathrm{B}_{t}, t \in[0,1]\right\}$ the following $\mathrm{P}$-almost-everywhere limit exists and

$$
\lim _{n \rightarrow \infty} \frac{1}{n} \sum_{k=0}^{n-1}\left(\mathrm{~B}_{\frac{k}{n}}\right)^{2}=\int_{0}^{1} \mathrm{~B}_{u}^{2} d u \quad \mathrm{P} \text { - a.e. }
$$

where the right-hand side integrale has to be understood path by path and with respect to Lebesgue measure on $[0,1]$. Besides, thanks to the theory of integration with respect to Brownian motion and since $\int_{0}^{1} \mathrm{E}\left[\mathrm{B}_{u}^{2}\right] d u=1 / 2<\infty$, the process $\left\{\mathrm{B}_{t}, t \in[0,1]\right\}$ is integrable with respect to the Brownian motion $\left\{\mathrm{B}_{t}^{\prime}, t \in[0,1]\right\}$ and the following in-probability limit exists

$$
\mathrm{P}-\lim _{n \rightarrow \infty} \sum_{k=0}^{n-1} \mathrm{~B}_{\frac{k}{n}}\left(\mathrm{~B}_{\frac{k+1}{n}}^{\prime}-\mathrm{B}_{\frac{k}{n}}^{\prime}\right)=\int_{0}^{1} \mathrm{~B}_{u} d \mathrm{~B}_{u}^{\prime}
$$


Hence

$\lim _{n \rightarrow \infty} \mathcal{L}_{\theta}\left[\left(\frac{1}{n^{2}} \sum_{k=1}^{n-1} S_{k-1}^{2}, \frac{1}{n} \sum_{k=1}^{n-1} Y_{k} S_{k-1}\right)\right]=\mathcal{L}\left[\left(G_{\theta}(P) \int_{0}^{1} \mathrm{~B}_{u}^{2} d u, \sqrt{G_{\theta}(P) H_{\theta}(P)} \int_{0}^{1} \mathrm{~B}_{u} d \mathrm{~B}_{u}^{\prime}\right)\right]$

and the convergence is uniform with respect to $\theta$ varying in any compact subset of $\mathbb{R}$. Then with relations (29) and (31), we deduce that

$$
\lim _{n \rightarrow \infty} \mathcal{L}_{\theta}\left[\frac{J_{n P+}}{n^{2}}, \frac{U_{n P+\cdot}}{n}\right]=\mathcal{L}\left[G_{\theta}(P) H_{\theta}(P) \int_{0}^{1} \mathrm{~B}_{u}^{2} d u, \sqrt{G_{\theta}(P) H_{\theta}(P)} \int_{0}^{1} \mathrm{~B}_{u} d \mathrm{~B}_{u}^{\prime}\right]
$$

in $\mathcal{C}[0, P] \times \mathcal{C}[0, P]$. This gives convergence (27) uniformly with respect to $\theta$ varying in any compact subset of $\mathbb{R}$.

\subsubsection{Proof of Theorem 4}

Here the true value of the parameter $\theta$ is equal to 0 and the function $f(\cdot)$ is non identically null. The observation is $\left\{\xi_{t}, t \in[0, T]\right\}=\left\{\xi_{0}+\mathrm{B}_{t}, t \in[0, T]\right\}$. With respect to the P-periodic Langevin model (1), the maximum likelihood estimator is still defined by relation (9). Following the same arguments as in the proof of Lemma 2, we obtain that

$$
J_{n P+t}=H_{0}(P) \sum_{k=1}^{n-1}\left(\sum_{j=0}^{k-1} Z_{j}\right)^{2}+o_{\mathrm{P}_{0}}\left(n^{2}\right)
$$

and

$$
U_{n P+t}=\sum_{k=1}^{n-1} \sum_{j=0}^{k-1} Y_{k} Z_{j}+o_{\mathrm{P}_{0}}(n)
$$

uniformly with respect to $t \in[0, P]$, where

$$
H_{0}(t)=\int_{0}^{t} f(u)^{2} d u, \quad Z_{j}=\mathrm{B}_{(j+1) P}-\mathrm{B}_{j P} \quad \text { and } \quad Y_{k}=\int_{k P}^{(k+1) P} f(u) d \mathrm{~B}_{u} .
$$

In contrast to the proof of Theorem 3 above, $F(P)$ is not here necessarily null and the random variables $Z_{j}$ and $Y_{j}$ are not necessarily independent, indeed $\operatorname{cov}_{0}\left[Y_{j}, Z_{j}\right]=\mathrm{E}_{0}\left[Y_{j} Z_{j}\right]=F(P)$ for any $j \in \mathbb{N}$. Since $\operatorname{var}_{0}\left[Z_{j}\right]=P$, the residual part of the linear regression of $Y_{j}$ on $Z_{j}$ is equal to $Y_{j}^{*}:=Y_{j}-\frac{F(P)}{P} Z_{j}$. Then the random vector $\left(Y_{0}^{*}, \ldots, Y_{n-1}^{*}, Z_{0}, \ldots, Z_{n-1}\right)$ is a Gaussian vector, its components are independent and $\operatorname{var}_{0}\left[Y_{j}^{*}\right]=H^{*}:=H_{0}(P)-\frac{F(P)^{2}}{P}$. Notice also that

$$
\sum_{k=1}^{n-1} \sum_{j=0}^{k-1} Y_{k} Z_{j}=\sum_{k=1}^{n-1} \sum_{j=0}^{k-1} Y_{k}^{*} Z_{j}+\frac{F(P)}{P} \sum_{k=1}^{n-1} \sum_{j=0}^{k-1} Z_{k} Z_{j}
$$

Now following the arguments of the proof of Lemma 2, consider $\left\{\mathrm{B}_{t}: t \in[0,1]\right\}$ and $\left\{\mathrm{B}_{t}^{\prime}: t \in[0,1]\right\}$ two independent Brownian motions. Let

$$
Y_{j}^{(0)}=\sqrt{n H^{*}}\left(\mathrm{~B}_{\frac{j+1}{n}}^{\prime}-\mathrm{B}_{\frac{j}{n}}^{\prime}\right) \quad \text { and } \quad Z_{j}^{(0)}=\sqrt{n P}\left(\mathrm{~B}_{\frac{j+1}{n}}-\mathrm{B}_{\frac{j}{n}}\right)
$$


for any $j=0, \ldots, n-1$. Then $\mathcal{L}_{0}\left[Y_{0}^{*}, \ldots, Y_{n-1}^{*}, Z_{0}, \ldots, Z_{n-1}\right]=\mathcal{L}\left[Y_{0}^{(0)}, \ldots, Y_{n-1}^{(0)}, Z_{0}^{(0)}, \ldots, Z_{n-1}^{(0)}\right]$ and the law of the random vector

$$
\left(\sum_{k=1}^{n-1}\left(\sum_{j=0}^{k-1} Z_{j}\right)^{2}, \sum_{k=1}^{n-1} \sum_{j=0}^{k-1} Y_{k}^{*} Z_{j}, \sum_{k=1}^{n-1} \sum_{j=0}^{k-1} Z_{k} Z_{j}\right)
$$

coincides with the law of

$$
\left(\sum_{k=1}^{n-1}\left(\sum_{j=0}^{k-1} Z_{j}^{(0)}\right)^{2}, \sum_{k=1}^{n-1} \sum_{j=0}^{k-1} Y_{k}^{(0)} Z_{j}^{(0)}, \sum_{k=1}^{n-1} \sum_{j=0}^{k-1} Z_{k}^{(0)} Z_{j}^{(0)}\right)
$$

thus with the law of

$$
\left(n P \sum_{k=1}^{n-1}\left(\mathrm{~B}_{\frac{k}{n}}\right)^{2}, n \sqrt{H^{*} P} \sum_{k=1}^{n-1}\left(\mathrm{~B}_{\frac{k+1}{n}}^{\prime}-\mathrm{B}_{\frac{k}{n}}^{\prime}\right) \mathrm{B}_{\frac{k}{n}}, n P \sum_{k=1}^{n-1}\left(\mathrm{~B}_{\frac{k+1}{n}}-\mathrm{B}_{\frac{k}{n}}\right) \mathrm{B}_{\frac{k}{n}}\right) .
$$

Thanks to convergences (32) and (33) as well as

$$
\lim _{n \rightarrow \infty} \sum_{k=1}^{n-1}\left(\mathrm{~B}_{\frac{k+1}{n}}-\mathrm{B}_{\frac{k}{n}}\right) \mathrm{B}_{\frac{k}{n}}=\int_{0}^{1} \mathrm{~B}_{u} d \mathrm{~B}_{u}=\frac{\mathrm{B}_{1}^{2}-1}{2} \quad \text { in probability }
$$

we deduce that

$$
\lim _{T \rightarrow \infty} \mathcal{L}_{0}\left[\left(\frac{J_{T}}{T^{2}}, \frac{U_{T}}{T}\right)\right]=\mathcal{L}\left[\left(\frac{H_{0}(P)}{P} \int_{0}^{1} \mathrm{~B}_{u}^{2} d u, \sqrt{\frac{H^{*}}{P}} \int_{0}^{1} \mathrm{~B}_{u} d \mathrm{~B}_{u}^{\prime}+\frac{F(P)}{P} \int_{0}^{1} \mathrm{~B}_{u} d \mathrm{~B}_{u}\right)\right] .
$$

where $H^{*}=H_{0}(P)-F(P)^{2} / P$. Thus

$$
\lim _{T \rightarrow \infty} \mathcal{L}_{0}\left[T \widehat{\theta}_{T}\right]=\mathcal{L}\left[\frac{\sqrt{P H_{0}(P)-F(P)^{2}} \int_{0}^{1} \mathrm{~B}_{u} d \mathrm{~B}_{u}^{\prime}+F(P) \int_{0}^{1} \mathrm{~B}_{u} d \mathrm{~B}_{u}}{H_{0}(P) \int_{0}^{1} \mathrm{~B}_{u}^{2} d u}\right] .
$$

Owing to independence between the random variable $\zeta$ defined by relation (28) and the Brownian motion $\left\{\mathrm{B}_{u}, u \in[0,1]\right\}$, we readily conclude the proof of Theorem 4 .

\subsubsection{Proof of Theorem 5}

First we study the asymptotic behaviour of the quadratic variation $\left\{J_{t}, t \geq 0\right\}$ of the martingale $\left\{U_{t}, t \geq 0\right\}$.

Lemma 3 If $\theta F(P)>0$, then

$$
\lim _{n \rightarrow \infty} e^{-2 n \theta F(P)} J_{n P+}=K_{\theta}(\cdot)^{2}\left(\xi_{0}+\zeta_{3}^{(\theta)}\right)^{2} \quad \mathrm{P}_{\theta} \text {-a.e. in } \mathcal{C}[0, P]
$$

where $K_{\theta}(\cdot)$ is defined in Theorem 5 and $\zeta_{3}^{(\theta)}$ is some real-valued Gaussian variable independent with respect to $\xi_{0}$. Furthermore

$$
\lim _{n \rightarrow \infty} \sup _{\theta \in K_{+}} \sup _{t \in[0, P]} \mathrm{E}_{\theta}\left[\left|e^{-2 n \theta F(P)} J_{n P+t}-K_{\theta}(t)^{2}\left(\xi_{0}+\zeta_{3}^{(\theta)}\right)^{2}\right|\right]=0
$$

for any compact set $K_{+} \subset\{\theta \in \mathbb{R}: \theta F(P)>0\}$. 
Proof According to limit (5) we know that

$$
\lim _{n \rightarrow \infty} e^{-2 n \theta F(P)} \int_{0}^{\cdot} f(u)^{2} \xi_{n P+u}^{2} d u=H_{\theta}(\cdot)\left(\xi_{0}+\zeta_{3}^{(\theta)}\right)^{2} \quad \mathrm{P}_{\theta^{-}} \text {a.e. in } \mathcal{C}[0, P]
$$

where $\zeta_{3}^{(\theta)}$ is some real-valued zero-mean Gaussian variable independent with respect to $\xi_{0}$, and with variance equal to $\frac{G_{\theta}(P)}{1-e^{2 \theta F(p)}}$. Besides $J_{n P}$ can be expressed as

$$
J_{n P}=\sum_{k=0}^{n-1} \int_{0}^{P} f(u)^{2} \xi_{k P+u}^{2} d u=\sum_{k=0}^{n-1} e^{2 k \theta F(P)} e^{-2 k \theta F(P)} \int_{0}^{P} f(u)^{2} \xi_{k P+u}^{2} d u
$$

for any integer $n>0$. Then Toeplitz lemma on series convergence implies the almost sure convergence $(34)$ since $\theta F(P)>0$.

To prove the uniform $\mathrm{L}^{1}\left(\mathrm{P}_{\theta}\right)$-convergence, let $K_{+}$be any compact subset of $\{\theta \in \mathbb{R}$ : $\theta F(P)>0\}$ and consider decomposition (21) which is also valid for $\theta F(P)>0$. As the random variables $Z_{j}$ s are independent with the same law $\mathcal{N}\left(0, G_{\theta}(P)\right)$, the hypothesis $\theta F(P)>0$ entails that $S_{n}$ converges in quadratic mean uniformly with respect to $\theta$ varying in $K_{+}$as $n \rightarrow \infty$. It is easy to see that the limit is equal to $\zeta_{3}^{(\theta)}$ defined above. So $e^{-2 n \theta F(P)} A_{4}=$ $e^{-2 n \theta F(P)} H_{\theta}(t)\left(\xi_{0}+S_{n}\right)^{2}$ converges in $\mathrm{L}^{1}\left(\mathrm{P}_{\theta}\right)$ uniformly with respect to $t \in[0, P]$ and to $\theta$ varying in $K_{+}$as $n \rightarrow \infty$ to $H_{\theta}(t)\left(\xi_{0}+\zeta_{3}^{(\theta)}\right)^{2}$. Furthermore, applying Toeplitz lemma on series convergence in $\mathrm{L}^{1}\left(\mathrm{P}_{\theta}\right)$ we deduce that $e^{-2 n \theta F(P)} A_{1}$ converges in $\mathrm{L}^{1}\left(\mathrm{P}_{\theta}\right)$ to

$$
\frac{H_{\theta}(P)}{e^{2 \theta F(P)}-1}\left(\xi_{0}+\zeta_{3}^{(\theta)}\right)^{2}
$$

uniformly with respect to $\theta \in K_{+}$as $n \rightarrow \infty$. Inequality (22) implies that $e^{-2 n \theta F(P)}\left(A_{2}+A_{5}\right)$ converges to 0 in $\mathrm{L}^{1}\left(\mathrm{P}_{\theta}\right)$ uniformly with respect to $t \in[0, P]$ and to $\theta \in K_{+}$as $n \rightarrow \infty$. Cauchy-Schwarz inequality involves that

$$
\left(\mathrm{E}_{\theta}\left[\left|\left(\xi_{0}+S_{n}\right) \mathbf{Z}_{n}(t)\right|\right]\right)^{2} \leq \mathrm{E}_{\theta}\left[\left|\xi_{0}+S_{n}\right|^{2}\right] \mathrm{E}_{\theta}\left[\left|\mathbf{Z}_{n}(t)\right|^{2}\right]
$$

and thanks to inequality (22) we obtain that $e^{-2 n \theta F(P)}\left(A_{3}+A_{6}\right)$ converge to 0 in $\mathrm{L}^{1}$ uniformly with $\theta$ varying in $K_{+}$as $n \rightarrow \infty$. Hence the proof of the lemma is achieved.

Remark that $J_{T}$ converges to infinity $\mathrm{P}_{\theta}$-a.e. in $\mathbb{R}$ as $T \rightarrow \infty$ when $\theta F(P)>0$. Then the central limit theorem for martingales (see e.g. Kutoyants 2004, Theorem 1.19) applies and $J_{T}^{1 / 2}\left(\widehat{\theta}_{T}-\theta\right)=J_{T}^{-1 / 2} U_{T}$ converges in law to the gaussian law $\mathcal{N}(0,1)$ as $T \rightarrow \infty$ for any $\theta$ such that $\theta F(P)>0$. However to get the uniform convergence we need to refine the analysis. In Lemma 3 we have obtained the convergence of $e^{-2 n \theta F(P)} J_{n P+}$. To prove Theorem 5 , first we go further into details for the asymptotic behaviour of $e^{-n \theta F(P)} U_{n P+t}$.

\section{Lemma 4}

$$
\lim _{n \rightarrow \infty} \mathcal{L}_{\theta}\left[e^{-n \theta F(P)} U_{n P+\cdot}\right]=\mathcal{L}\left[\left(\xi_{0}+\zeta_{3}^{(\theta)}\right)\left(\zeta_{4}^{(\theta)}+\mathbf{Y}^{(\theta)}(\cdot)\right)\right] \quad \text { in } \quad \mathcal{C}[0, P]
$$


uniformly with respect to $\theta$ in any compact subset of $\{\theta \in \mathbb{R}: \theta F(P)>0\}$. Here $\zeta_{3}^{(\theta)}$ and $\zeta_{4}^{(\theta)}$ are two real-valued zero-mean Gaussian variables with variances

$$
\operatorname{var}\left[\zeta_{3}^{(\theta)}\right]=\frac{G_{\theta}(P)}{1-e^{-2 \theta F(P)}} \quad \text { respectively } \quad \operatorname{var}\left[\zeta_{4}^{(\theta)}\right]=\frac{H_{\theta}(P)}{e^{2 \theta F(P)}-1},
$$

and the process $\mathbf{Y}^{(\theta)}:=\left\{\mathbf{Y}^{(\theta)}(t), t \in[0, P]\right\}$ is Gaussian and admits the representation

$$
\mathbf{Y}^{(\theta)}(t)=\int_{0}^{t} f(u) e^{\theta F(u)} d \mathrm{~B}_{u}^{\prime}
$$

for some Brownian motion $\left\{\mathrm{B}_{t}^{\prime}, t \in[0, P]\right\}$. Moreover $\xi_{0}, \zeta_{3}^{(\theta)}, \zeta_{4}^{(\theta)}$ and $\mathbf{Y}^{(\theta)}$ are independent.

Proof The scheme of the proof we follow is inspired by the proof presented by Kutoyants (2004, Proposition 3.46) when the modulation function $f(\cdot)$ is identically equal to 1 (see also Basawa and Scott 1983). Mainly let us express $U_{T}, T>0$, as

$$
U_{T}=\xi_{0} \psi_{T}+\int_{0}^{T} M_{u} d \psi_{u}
$$

where $\left\{\psi_{t}, t \geq 0\right\}$ is the Gaussian process defined by

$$
\psi_{t}:=\int_{0}^{t} f(u) e^{\theta F(u)} d \mathrm{~B}_{u}
$$

and $\left\{M_{t}, t \geq 0\right\}$ is the martingale process defined by $M_{t}:=e^{\theta F(t)} \xi_{t}-\xi_{0}$. Thanks to Itô formula we have

$$
U_{T}=\left(\xi_{0}+M_{T}\right) \psi_{T}-\int_{0}^{T} \psi_{u} d M_{u}-\frac{1}{2}[\psi, M]_{T},
$$

the quadratic covariation $[\psi, M]_{T}$ being equal to

$$
[\psi, M]_{T}=\int_{0}^{T} f(u) e^{-\theta F(u)} e^{\theta F(u)} d u=\int_{0}^{T} f(u) d u=F(T)
$$

Notice that $\psi_{n P+t}$ is zero-mean and its variance is equal to

$$
\mathrm{E}\left[\psi_{n P+t}^{2}\right]=\int_{0}^{n P+t} f(u)^{2} e^{2 \theta F(u)} d u=\frac{\left(e^{2 n \theta F(P)}-1\right) H_{\theta}(P)}{e^{2 \theta F(P)}-1}+e^{2 n \theta F(P)} H_{\theta}(t)
$$

for any $n \in \mathbb{N}$ and any $t \in[0, P]$.

Now we are going to study the asymptotic behaviour of each term of expression (35). Let $K_{+}$any compact subset of $\{\theta \in \mathbb{R}: \theta F(P)>0\}$.

(i) The periodicity of the function $f(\cdot)$ implies that

$$
\lim _{n \rightarrow \infty} \frac{1}{n}[\psi, M]_{n P+t}=F(P)
$$


uniformly with respect to $t \in[0, P]$ and $\theta \in K_{+}$.

(ii) The second term of the right hand side of expression (35) can be decomposed as

$$
\begin{aligned}
\int_{0}^{T} \psi_{u} d M_{u} & =\int_{0}^{n P} \psi_{u} d M_{u}+\int_{n P}^{n P+t} e^{-\theta F(u)} \psi_{u} d \mathrm{~B}_{u} \\
& =\int_{0}^{n P} \psi_{u} d M_{u}+e^{-n \theta F(P)} \mathbf{V}_{n}(t),
\end{aligned}
$$

the $\mathcal{C}[0, P]$-valued random sequence $\left(\mathbf{V}_{n}\right)_{n \in \mathbb{N}}$ being defined by

$$
\mathbf{V}_{n}(t):=\int_{0}^{t} e^{-\theta F(u)} \psi_{n P+u} d \mathrm{~B}_{u}^{(n P)}
$$

The variance of the first term of the right hand side of equality (37) is equal to

$$
\mathrm{E}_{\theta}\left[\left(\int_{0}^{n P} \psi_{u} d M_{u}\right)^{2}\right]=\mathrm{E}_{\theta}\left[\left(\int_{0}^{n P} e^{-\theta F(u)} \psi_{u} d \mathrm{~B}_{u}\right)^{2}\right]=\int_{0}^{n P} e^{-2 \theta F(u)} \mathrm{E}_{\theta}\left[\psi_{u}^{2}\right] d u .
$$

In the following using again the fact that $F(k P+u)=k F(P)+F(u)$, and replacing $\mathrm{E}_{\theta}\left[\psi_{k P+u}^{2}\right]$ by its expression (36), we obtain

$$
\begin{aligned}
\int_{0}^{n P} e^{-2 \theta F(u)} \mathrm{E}_{\theta}\left[\psi_{u}^{2}\right] d u & =\sum_{k=0}^{n-1} \int_{0}^{P} e^{-2 \theta(k F(P)+F(u))} \mathrm{E}_{\theta}\left[\psi_{k P+u}^{2}\right] d u \\
& =\frac{H_{\theta}(P) G_{\theta}(P)}{e^{2 \theta F(P)}-1}\left(n-\frac{1-e^{-2 n \theta F(P)}}{1-e^{-2 \theta F(P)}}\right)+n \int_{0}^{P} H_{\theta}(u) e^{-2 \theta F(u)} d u .
\end{aligned}
$$

Hence

$$
\lim _{n \rightarrow \infty} \frac{1}{n} \int_{0}^{n P} e^{-2 \theta F(u)} \mathrm{E}_{\theta}\left[\psi_{u}^{2}\right] d u=\frac{H_{\theta}(P) G_{\theta}(P)}{e^{2 \theta F(P)}-1}+\int_{0}^{P} H_{\theta}(u) e^{-2 \theta F(u)} d u
$$

and $\frac{1}{n} \int_{0}^{n P} \psi_{u} d M_{u}$ converges to 0 in $\mathrm{P}_{\theta}$-quadratic mean uniformly with respect to $\theta \in K_{+}$as $n \rightarrow \infty$.

As for the second term of the right hand side of equality (37), notice that for each integer $n$ we have

$$
\mathbf{V}_{n}(t)=\mathbf{V}_{n}(s)+\psi_{n P+s} \int_{s}^{t} e^{-\theta F(u)} d \mathrm{~B}_{u}^{(n P)}+\int_{s}^{t} e^{-\theta F(u)}\left(\int_{n P+s}^{n P+u} f(v) e^{\theta F(v)} d \mathrm{~B}_{v}\right) d \mathrm{~B}_{u}^{(n P)}
$$

when $0 \leq s \leq t \leq P$, which entails that the process $\mathbf{V}_{n}:=\left\{\mathbf{V}_{n}(t), t \in[0, P]\right\}$ is a continuous martingale with respect to the filtration $\left\{\mathcal{F}_{t}^{(n P)}: 0 \leq t \leq P\right\}$ generated by $\mathrm{B}^{(n P)}=\left\{\mathrm{B}_{t}^{(n P)}, t \in\right.$ $[0, P]\}$. It is zero-mean, and by equality (36) it verifies

$$
\begin{aligned}
\mathrm{E}_{\theta}\left[\mathbf{V}_{n}(t)^{2}\right] & =\int_{0}^{t} e^{-2 \theta F(u)} \mathrm{E}_{\theta}\left[\psi_{n P+u}^{2}\right] d u \\
& =\frac{\left(e^{2 n \theta F(P)}-1\right) H_{\theta}(P) G_{\theta}(t)}{e^{2 \theta F(P)}-1}+e^{2 n \theta F(P)} \int_{0}^{t} e^{-2 \theta F(u)} H_{\theta}(u) d u .
\end{aligned}
$$


Doob maxima inequality for continuous martingales implies that

$$
\mathrm{P}_{\theta}\left[\max _{t \in[0, P]}\left|\mathbf{V}_{n}(t)\right|>\epsilon\right] \leq \frac{\mathrm{E}_{\theta}\left[\mathbf{V}_{n}(P)^{2}\right]}{\epsilon^{2}}
$$

thus

$$
\mathrm{P}_{\theta}\left[e^{-n \theta F(P)} \max _{t \in[0, P]}\left|\mathbf{V}_{n}(t)\right|>\epsilon\right] \leq \frac{1}{\epsilon^{2}}\left(\frac{H_{\theta}(P) G_{\theta}(P)}{e^{2 \theta F(P)}-1}+\int_{0}^{t} e^{-2 \theta F(u)} H_{\theta}(u) d u\right)
$$

and

$$
\lim _{n \rightarrow \infty} \frac{e^{-n \theta F(P)}}{n} \mathbf{V}_{n}=0 \quad \text { in } \mathrm{P}_{\theta} \text {-probability in } \mathcal{C}[0, P]
$$

uniformly with respect to $\theta \in K_{+}$. Hence

$$
\lim _{n \rightarrow \infty} \frac{1}{n} \int_{0}^{n P+\cdot} \psi_{u} d M_{u}=0 \quad \text { in } \mathrm{P}_{\theta} \text {-probability in } \mathcal{C}[0, P]
$$

uniformly with respect to $\theta \in K_{+}$.

(iii) By the definition of the process $\left\{\psi_{t}, t \geq 0\right\}$, and by the periodicity of the function $f(\cdot)$, we have

$$
\psi_{n P+t}=\int_{0}^{n P} f(u) e^{\theta F(u)} d \mathrm{~B}_{u}+e^{n \theta F(P)} \int_{0}^{t} f(u) e^{\theta F(u)} d \mathrm{~B}_{u}^{(n P)},
$$

the two terms being Gaussian and independent. We readily deduce that

$$
\lim _{n \rightarrow \infty} \mathcal{L}_{\theta}\left[e^{-n \theta F(P)} \psi_{n P+} \cdot\right]=\mathcal{L}\left[\zeta_{4}^{(\theta)}+\mathbf{Y}^{(\theta)}(\cdot)\right] \quad \text { in } \mathcal{C}[0, P]
$$

uniformly with respect to $\theta \in K_{+}$where $\zeta_{4}^{(\theta)}$ is a real-valued Gaussian variable with zero-mean and variance equal to $H_{\theta}(P)\left(e^{2 \theta F(P)}-1\right)^{-1}$, and the $P$-segment process $\mathbf{Y}^{(\theta)}:=\left\{\mathbf{Y}^{(\theta)}(t), t \in\right.$ $[0, P]\}$ is Gaussian and admits the following representation

$$
\mathbf{Y}^{(\theta)}(t)=\int_{0}^{t} f(u) e^{\theta F(u)} d \mathrm{~B}_{u}^{\prime}
$$

for some Brownian motion $\left\{\mathrm{B}_{t}^{\prime}, t \in[0, P]\right\}$ such that the random variable $\zeta_{4}^{(\theta)}$ and the process $\mathbf{Y}^{(\theta)}$ are independent.

(iv) Following the proof of limit (5) (see Dehay 2014) we can see that $M_{T}$ converges in $\mathrm{P}_{\theta^{-}}$ probability to some Gaussian variable $\zeta_{3}^{(\theta)}$ as $T \rightarrow \infty$, uniformly with respect to $\theta \in K_{+}$. So it remains to establish the joint convergence of the terms of expression (35), and the independence between the different random limits to conclude the proof of Lemma 4 .

First recall that by hypothesis the initiale value $\xi_{0}$ is independent with respect to the Brownian motion $\left\{\mathrm{B}_{t}, t \geq 0\right\}$. Now let $n_{1}<n$. Since

$$
M_{n_{1} P+t}=\int_{0}^{n_{1} P+t} e^{-\theta F(u)} d \mathrm{~B}_{u} \quad \text { and } \quad \psi_{n P+t}-\psi_{\left(n_{1}+1\right) P}=\int_{\left(n_{1}+1\right) P}^{n P+t} f(u) e^{\theta F(u)} d \mathrm{~B}_{u}
$$


for any $t \in[0, P]$, the random variables $\xi_{0}, \psi_{n P}-\psi_{\left(n_{1}+1\right) P}$, and the processes $\left\{M_{n_{1} P+t}, t \in\right.$ $[0, P]\},\left\{\psi_{n P+t}-\psi_{n P}, t \in[0, P]\right\}$ are independent. Consequently, thanks to the convergence results for $M_{T}$ and $\psi_{T}$, the law

$$
\mathcal{L}_{\theta}\left[\left(\xi_{0}, M_{\cdot+n_{1} P}, e^{-n \theta F(P)}\left(\psi_{n P}-\psi_{\left(n_{1}+1\right) P}\right), e^{-n \theta F(P)}\left(\psi_{n P+}-\psi_{n P}\right)\right)\right]
$$

converges to the law $\mathcal{L}\left[\left(\xi_{0}, \zeta_{3}^{(\theta)}, \zeta_{4}^{(\theta)}, \mathbf{Y}^{(\theta)}(\cdot)\right)\right]$ in $\mathbb{R} \times \mathcal{C}[0, P] \times \mathbb{R} \times \mathcal{C}[0, P]$, as $n_{1}$ and $n$ go to infinity as well as $n-n_{1}$. We deduce that the random variables $\xi_{0}, \zeta_{3}^{(\theta)}, \zeta_{4}^{(\theta)}$ and the process $\left\{\mathbf{Y}^{(\theta)}(t), t \in[0, P]\right\}$ are independent. Furthermore from expression (36), we easily verify that $e^{-n \theta F(P)} \psi_{\left(n_{1}+1\right) P}$ converges in $\mathrm{P}_{\theta}$-quadratic mean to 0 uniformly with respect to $\theta \in K_{+}$as $n-n_{1}$ goes to infinity. Then thanks to Slutsky lemma, we readily deduce that

$$
\lim _{n \rightarrow \infty} \mathcal{L}_{\theta}\left[\left(\xi_{0}, M_{n P+}, e^{-n \theta F(P)} \psi_{n P+}\right)\right]=\mathcal{L}\left[\left(\xi_{0}, \zeta_{3}^{(\theta)}, \zeta_{4}^{(\theta)}+\mathbf{Y}^{(\theta)}(\cdot)\right)\right]
$$

uniformly with respect to $\theta \in K_{+}$as $n \rightarrow \infty$. Finally, owing to Itô formula (35) and the convergence results of the previous parts (i), (ii) and (iii), Lemma 4 is proved.

\section{Proof of Theorem 5 Since}

$$
e^{n \theta F(P)}\left(\widehat{\theta}_{n P+t}-\theta\right)=\frac{e^{-n \theta F(P)} U_{n P+t}}{e^{-2 n \theta F(P)} J_{n P+t}},
$$

it suffices to establish the convergence of $\mathcal{L}\left(e^{-2 n \theta F(P)} J_{n P+t}, e^{-n \theta F(P)} U_{n P+t}\right)$ in $\mathcal{C}[0, P] \times \mathcal{C}[0, P]$ as $n \rightarrow \infty$, to prove Theorem 5 . For this purpose we follow the idea of part (iv) of the proof of Lemma 4 , and we use the same notations. Let $n_{1}<n$. By definition

$$
M_{n_{1} P+t}=\int_{0}^{n_{1} P+t} e^{-\theta F(u)} d \mathrm{~B}_{u} \quad \text { and } \quad J_{n_{1} P+t}=\int_{0}^{n_{1} P+t} f(u)^{2} \xi_{u}^{2} d \mathrm{~B}_{u} .
$$

The independence between the processes $\left\{\xi_{n_{1} P+t}, t \in[0, P]\right\}$ and $\left\{\mathrm{B}_{\left(n_{1}+1\right) P+t}-\mathrm{B}_{\left(n_{1}+1\right) P}, t \geq 0\right\}$ implies that the random variable $\psi_{n P}-\psi_{\left(n_{1}+1\right) P}$ and the processes $\left\{\left(M_{n_{1} P+t}, J_{n_{1} P+t}\right), t \in\right.$ $[0, P]\},\left\{\psi_{n P+t}-\psi_{n P}, t \in[0, P]\right\}$ are independent. According to the convergence results for $M_{n P+t}, J_{n P+t}$ and $\psi_{n P+t}$, the law

$$
\mathcal{L}_{\theta}\left[\left(\xi_{0}, M_{n P+}, e^{-2 n \theta F(P)} J_{n P+}, e^{-n \theta F(P)} \psi_{n P+} \cdot\right)\right]
$$

converges to $\mathcal{L}\left[\left(\xi_{0}, \zeta_{3}^{(\theta)}, K_{\theta}(\cdot)^{2}\left(\xi_{0}+\zeta_{3}^{(\theta)}\right)^{2}, \zeta_{4}^{(\theta)}+\mathbf{Y}^{(\theta)}(\cdot)\right)\right]$ in $\mathbb{R} \times \mathcal{C}[0, P] \times \mathcal{C}[0, P] \times \mathcal{C}[0, P]$.

Hence

$\lim _{n \rightarrow \infty} \mathcal{L}_{\theta}\left[\left(e^{-2 n \theta F(P)} J_{n P+\cdot}, e^{-n \theta F(P)} U_{n P+}\right)\right]=\mathcal{L}\left[K_{\theta}(\cdot)^{2}\left(\xi_{0}+\zeta_{3}^{(\theta)}\right)^{2},\left(\xi_{0}+\zeta_{3}^{(\theta)}\right)\left(\zeta_{4}^{(\theta)}+\mathbf{Y}^{(\theta)}(\cdot)\right)\right]$

in $\mathcal{C}[0, P] \times \mathcal{C}[0, P]$. The convergences being uniform with respect to $\theta$ varying in any compact subset of $\{\theta \in \mathbb{R}: \theta F(P)>0\}$. As $\mathrm{P}\left[\xi_{0}+\zeta_{3}^{(\theta)}=0\right]=0$, we readily deduce that

$$
\lim _{n \rightarrow \infty} \mathcal{L}_{\theta}\left[e^{n \theta F(P)}\left(\widehat{\theta}_{n P+}-\theta\right)\right]=\mathcal{L}\left[\frac{\zeta_{4}^{(\theta)}+\mathbf{Y}^{(\theta)}(\cdot)}{K_{\theta}(\cdot)\left(\xi_{0}+\zeta_{3}^{(\theta)}\right)}\right] \quad \text { in } \mathcal{C}[0, P]
$$


uniformly with respect to $\theta$ varying in the compact set $K_{+}$.

For any $t \in[0, P]$ the random variables $\zeta_{4}^{(\theta)}$ and $\mathbf{Y}^{(\theta)}(t)$ are Gaussian independent, then we deduce that $K_{\theta}(t)=\sqrt{\operatorname{var}\left[\zeta_{4}^{(\theta)}+\mathbf{Y}^{(\theta)}(t)\right]}$ and the random variable $\left(\zeta_{4}^{(\theta)}+\mathbf{Y}^{(\theta)}(t)\right) / K_{\theta}(t)$ is Gaussian $\mathcal{N}(0,1)$ and independent with respect to $\xi_{0}$ and $\zeta_{3}^{(\theta)}$. Then the proof of the theorem can readily be achieved.

\subsection{Proofs of results in Section 4}

\subsubsection{Proofs of Theorems 6 and 7}

The limit law in Theorem 2 (respectively Theorem 3) is absolutely continuous with respect to Lebesgue measure, and the set of points of discontinuity of each loss function $L$ in $\mathcal{L}$ is almost countable. Hence Theorem 6 (respectively Theorem 7) is a direct consequence of Theorem 2 (respectively Theorem 3) and the convergence result from (Ibragimov and Has'minskir 1981, Appendix I Theorem 8). Notice that the convergence result in (Ibragimov and Has'minskiĩ 1981) is uniform with respect to $\theta \in \Theta$.

\subsubsection{Proof of Theorem 8}

In order to evaluate the local asymptotic lower bound for the quadratic risk, we first study the asymptotic behaviour of $\mathrm{E}_{\theta}\left[J_{T}\right] / T^{2}$

Lemma 5 If $F(P)=0$ and $f(\cdot)$ is not identically null, then

$$
\lim _{T \rightarrow \infty} \frac{\mathrm{E}_{\theta}\left[J_{T}\right]}{T^{2}}=\frac{G_{\theta}(P) H_{\theta}(P)}{2 P^{2}}
$$

uniformly with respect to $\theta$ varying in any compact subset of $\mathbb{R}$.

Proof We know that

$$
\mathrm{E}_{\theta}\left[J_{n P+t}\right]=\int_{0}^{n P+t} f(u)^{2} \mathrm{E}_{\theta}\left[\xi_{u}^{2}\right] d u=\mathrm{E}_{\theta}\left[J_{n P}\right]+\int_{0}^{t} f(u)^{2} \mathrm{E}_{\theta}\left[\xi_{n P+u}^{2}\right] d u
$$

for all $n \in \mathbb{N}$ and $t \in[0, P]$. As $F(P)=0, F(n P+t)=F(t)$ we have $\mathrm{E}_{\theta}\left[\xi_{n P+t}^{2}\right]=e^{2 F(t)}\left(\mathrm{E}_{\theta}\left[\xi_{0}^{2}\right]+\right.$ $\left.n G_{\theta}(P)+G_{\theta}(t)\right)$. Thus

$$
\begin{aligned}
\mathrm{E}_{\theta}\left[J_{n P}\right] & =\int_{0}^{P} f(u)^{2} \sum_{k=0}^{n-1} \mathrm{E}_{\theta}\left[\xi_{k P+u}^{2}\right] d u \\
& =n \mathrm{E}_{\theta}\left[\xi_{0}^{2}\right] H_{\theta}(P)+\frac{n(n-1)}{2} H_{\theta}(P) G_{\theta}(P)+n \int_{0}^{P} f(u)^{2} e^{2 \theta F(u)} G_{\theta}(u) d u .
\end{aligned}
$$

Consequently

$$
\lim _{n \rightarrow \infty} \frac{\mathrm{E}_{\theta}\left[J_{n P}\right]}{n^{2}}=\frac{1}{2} H_{\theta}(P) G_{\theta}(P)
$$


and the lemma is proved.

Now we can state the asymptotic lower bound (18) for the mean square risk using van Trees inequality.

Let $\epsilon>0$ and $p_{\epsilon}(\cdot)$ be a density probability function with support in $[\theta-\epsilon, \theta+\epsilon]$ and which is continuously differentiable in $\mathbb{R}$. We know that $\mathrm{E}_{\theta}\left[U_{T}\right]=0, \mathrm{E}_{\theta}\left[U_{T}^{2}\right]=\mathrm{E}_{\theta}\left[J_{T}\right]<\infty$ and that the log-likelihood ratio $\Lambda_{T}^{(\theta)}$ of $\mathrm{P}_{\theta}^{T}$ to $\mathrm{P}_{0}^{T}$, satisfies $\frac{\partial}{\partial \theta} \Lambda_{T}^{(\theta)}(\xi)=U_{T}$ when $\xi=\xi^{(\theta)}$. Thus $\mathrm{E}_{\theta}\left[\frac{\partial}{\partial \theta} \Lambda_{T}^{(\theta)}(\xi)^{2}\right]<\infty$ and $\mathrm{E}_{\theta}\left[\frac{\partial}{\partial \theta} \Lambda_{T}^{(\theta)}(\xi)\right]=0$. Then for any real-valued random variable $\bar{\theta}_{T}$ measurable with respect to the observation $\left\{\xi_{t}, t \in[0, T]\right\}$, van Trees inequality (see Gill and Levit 1995) entails that

$$
\int_{\theta-\epsilon}^{\theta+\epsilon} \mathrm{E}_{\theta^{\prime}}\left[\left(\bar{\theta}_{T}-\theta^{\prime}\right)^{2}\right] p_{\epsilon}\left(\theta^{\prime}\right) d \theta^{\prime} \geq\left(\int_{\theta-\epsilon}^{\theta+\epsilon} \mathrm{E}_{\theta^{\prime}}\left[J_{T}\right] p_{\epsilon}\left(\theta^{\prime}\right) d \theta^{\prime}+I\left(p_{\epsilon}\right)\right)^{-1}
$$

where $I\left(p_{\epsilon}\right)$ is the Fisher information for a location parameter in $p_{\epsilon}(\cdot)$

$$
I\left(p_{\epsilon}\right)=\int_{\theta-\epsilon}^{\theta+\epsilon}\left(\frac{d}{d \theta^{\prime}} \ln p_{\epsilon}\left(\theta^{\prime}\right)\right)^{2} p_{\epsilon}\left(\theta^{\prime}\right) d \theta^{\prime}=\int_{\theta-\epsilon}^{\theta+\epsilon} \frac{\left(\frac{d}{d \theta^{\prime}} p_{\epsilon}\left(\theta^{\prime}\right)\right)^{2}}{p_{\epsilon}\left(\theta^{\prime}\right)} d \theta^{\prime}
$$

Thus

$$
\inf _{\bar{\theta}_{T}} \sup _{\left|\theta^{\prime}-\theta\right|<\epsilon} \mathrm{E}_{\theta^{\prime}}\left[T^{2}\left(\bar{\theta}_{T}-\theta^{\prime}\right)^{2}\right] \geq\left(\int_{\theta-\epsilon}^{\theta+\epsilon} \mathrm{E}_{\theta^{\prime}}\left[\frac{J_{T}}{T^{2}}\right] p_{\epsilon}\left(\theta^{\prime}\right) d \theta^{\prime}+\frac{I\left(p_{\epsilon}\right)}{T^{2}}\right)^{-1} .
$$

From Lemma 5, we deduce that

$$
\begin{aligned}
\liminf _{T \rightarrow \infty} \inf _{\bar{\theta}_{T}} \sup _{\left|\theta^{\prime}-\theta\right|<\epsilon} \mathrm{E}_{\theta^{\prime}}\left[T^{2}\left(\bar{\theta}_{T}-\theta^{\prime}\right)^{2}\right] & \geq \lim _{T \rightarrow \infty}\left(\int_{\theta-\epsilon}^{\theta+\epsilon} \mathrm{E}_{\theta^{\prime}}\left[\frac{J_{T}}{T^{2}}\right] p_{\epsilon}\left(\theta^{\prime}\right) d \theta^{\prime}\right)^{-1} \\
& =\left(\int_{\theta-\epsilon}^{\theta+\epsilon} \frac{G_{\theta^{\prime}}(P) H_{\theta^{\prime}}(P)}{2 P^{2}} p_{\epsilon}\left(\theta^{\prime}\right) d \theta^{\prime}\right)^{-1}
\end{aligned}
$$

With $\epsilon \rightarrow 0$, we deduce the lower bound for the mean square deviation (18).

Thanks to the uniform convergence in $\theta$ of $T\left(\widehat{\theta}_{T}-\theta\right)$ in Theorem 3 and to (Ibragimov and Has'minskiĩ 1981, Appendix I Theorem 8), we readily deduce the second assertion of Theorem 8.

\subsubsection{Proof of Theorem 9}

Since the random variables $\xi_{0}, \zeta$ and $\zeta_{3}^{(\theta)}$ are independent and the random variables $\zeta$ and $\zeta_{3}^{(\theta)}$ are Gaussian, the limit law in Theorem 5 is absolutely continuous with respect to Lebesgue measure. Hence by the uniform convergence in law in Theorem 5, the convergence theorem of Ibragimov and Has'minskiř (1981, Appendix I Theorem 8) entails that for each $\theta$ such that $\theta F(P)>0$

$$
\lim _{n \rightarrow \infty} \sup _{\left|\theta-\theta^{\prime}\right|<M e^{-n \theta F(P)}}\left|\mathrm{E}_{\theta^{\prime}}\left[L\left(e^{n \theta^{\prime} F(P)}\left(\widehat{\theta}_{n P+t}-\theta^{\prime}\right)\right)\right]-\mathrm{E}\left[L\left(\frac{\zeta}{K_{\theta^{\prime}}(t)\left(\xi_{0}+\zeta_{3}^{\left(\theta^{\prime}\right)}\right)}\right)\right]\right|=0
$$


for any $t \in[0, P]$ and any $M>0$. Thanks to the continuity with respect to $\theta$ of $K_{\theta}(t)$ and of the law of $\zeta_{3}^{(\theta)}$ we readily deduce that the MLE $\widehat{\theta}_{n P+t}$ is locally asymptotically minimax for any $t \in[0, P]$.

\section{Acknowledgements}

The author would like to thank two anonymous referees for their careful reading of this manuscript and very valuable comments, as well as Pr. Yury Kutoyants for helpful discussions.

\section{References}

Antoni J. (2009) Cyclostationarity by examples, Mechan. System. Signal Process. 23, 987-1036. Barczy M. and Pap G. (2010) Asymptotic behaviour of maximum likelihood estimator for time inhomogeneous diffusion processes, Journal of Statistical Planning and Inference 140, 15761593.

Bishwal J.P. N. (2008) Parameter Estimation in Stochastic Differential Equations, SpringerVerlag, Berlin.

Basawa I.V. and Scott D.J. (1983) Asymptotic Optimal Inference for Non-ergodic Models, Lectures Notes in Statistics 17, Springer-Verlag, New-York.

Brown B.M. and Hewitt J.I. (1975) Asymptotic likelihood theory for diffusion processes, $J$. Appl. Prob. 12, 228-238.

Chaari F., Leśkow J., Napolitano A. and Sanchez-Ramirez A. (eds) (2014) Cyclostationarity : Theory and Methods, Lecture Notes in Mechanical Engineering, Springer-Verlag, Cham.

Collet P. and Martinez S. (2008) Asymptotic velocity of one dimensional diffusions with periodic drift, J. Math. Biol. 56, 765-792.

Davies R.B. (1985) Asymptotic inference when the amount of information is random : In Le Cam L. and Olshen R. (eds), Proceedings of the Berkeley Symposium in Honour of J. Neyman and J. Kiefer, vol. 2, Wadsworth, Belmont, 841-864.

Dehay D. (2014) Time-periodic-modulated-drift Langevin type stochastic differential equations, submitted

Dehling H., Franke B. and Kott T. (2010) Drift estimation for a periodic mean reversion process, Statist. Inference Stoch. Process. 13, 175-192.

Feigin P.D. (1976) Maximum likelihood estimation for continuous time stochastic processes, Adv. Appl. Prob. 8, 712-736.

Feigin P.D. (1979) Some comments concerning a curious singularity, J. Appl. Prob. 16, 440-444. 
Gardner W.A., Napolitano A. and Paura L. (2006) Cyclostationarity : half a century of research, Signal Processing 86, 639-697.

Gill R.D. and Levit B.Y. (1995) Applications of the van Trees inequality : a Bayesian CramérRao bound, Bernoulli 1, 59-79.

Gladyshev E.G. (1963) Periodically and almost periodically correlated processes, Theory Probab. Appl. 8, 173-177.

Hájek J. (1972) Local asymptotic minimax and admissibility in estimation, in Proc. 6-th Berkeley Symposium Math. Statist. Probab., vol 1, 175-194, Univ. California Press.

Has'minskiǐ (1980) Stochastic Stability of Differential Equations, Sijthoff \& Noordhoff, Alphen aan den Rijn.

Hurd H.L. and Miamee A. (2007) Periodically Correlated Random Sequences : Spectral Theory and Pratice, J. Wiley \& Sons, Hoboken.

Höpfner R. and Kutoyants Y. (2010) Estimating discontinuous periodic signals in a time inhomogeneous diffusion, Statist. Inference Stoch. Process. 13, 193-230.

Ibragimov I.A. and Has'minskiǔ R.Z. (1981) Statistical Estimation : Asymptotic Theory, SpringerVerlag, New-York.

Jeganathan P. (1982) On the asymptotic theory of estimation when the limit of the likelihood is mixed normal, Sankhia Ser. A 44, 173-212.

Jeganathan P. (1995) Some aspect of asymptotic theory with applications to time series models, Econometric Theory 11, 818-887.

Kutoyants Yu. A. (2004) Statistical Inference for Ergodic Diffusion Processes, Springer-Verlag, London.

Le Cam L. (1969) Théorie Asymptotique de la décision statistique, Univ. of Montréal Press, Montréal.

Le Cam L. (1986) Asymptotics Methods in Statistical Decision Theory, Springer-Verlag, NewYork.

Le Cam L. and Yang G.L. (1990) Asymptotics in Statistics : Some Basic Concepts, SpringerVerlag, New-York.

Liptser R. and Shiryaev A. (2001) Statistics of Random Processes, vol. I+II, (2nd edition) Springer-Verlag, New-York.

Meyn S and Tweedie R.L. (2009) Markov Chains and Stochastic Stability (2nd ed.), Cambridge University Press, Cambridge.

Mishra M.N. and Prakasa Rao B.L.S. (1985) Asymptotic study of the maximum likelihood estimator for non-homgeneous diffusion processes, Statistics and Decisions 3, 193-203. 
Phillips P.C.B. (1987) Towards a unified asymptotic theory for autoregression, Biometrika 74 (3), 535-547.

Revuz D. (1984) Markov Chains (2nd ed.), Elsevier Sciences Publishers, Amsterdam.

Revuz D. and Yor M. (1994) Continuous Martingales and Brownian motion (2nd ed.), SpringerVerlag, Berlin.

Serpedin E., Panduru F., Sari I. and Giannakis G.B. (2005) Bibliography on cyclostationarity, Signal Processing 85, 2233-2303. 